TRANSACTIONS OF THE

AMERICAN MATHEMATICAL SOCIETY

Volume 356, Number 7, Pages 2663-2684

S 0002-9947(03)03438-X

Article electronically published on October 8, 2003

\title{
SUMS OF SQUARES IN REAL RINGS
}

\author{
JOSÉ F. FERNANDO, JESÚS M. RUIZ, AND CLAUS SCHEIDERER
}

\begin{abstract}
Let $A$ be an excellent ring. We show that if the real dimension of $A$ is at least three then $A$ has infinite Pythagoras number, and there exists a positive semidefinite element in $A$ which is not a sum of squares in $A$.
\end{abstract}

\section{INTRODUCTION}

In the study of positive semidefinite $(=p s d)$ elements and sums of squares, the two main problems are these:

- Qualitative problem: To decide whether every positive semidefinite element is a sum of squares.

- Quantitative problem: To decide whether there is $p \in \mathbb{N}$ such that every sum of squares is a sum of $p$ squares, and to estimate the smallest such $p$.

These two problems have a meaning over any commutative ring $A$ : The set $\mathcal{P}(A)$ of psd elements of $A$ consists of all $f \in A$ which satisfy $\varphi(f) \geq 0$ for every homomorphism $\varphi: A \rightarrow k$ into an ordered (or real closed) field $k$. Clearly, $\mathcal{P}(A)$ contains $\Sigma(A)$, the set of sums of squares in $A$, and the qualitative problem is whether $\mathcal{P}(A)=\Sigma(A)$. The quantitative problem concerns the Pythagoras number, which is the smallest integer $p(A)=p \geq 1$ such that any sum of squares in $A$ is a sum of $p$ squares. One puts $p(A)=\infty$ if such an integer does not exist. The Pythagoras number is a very delicate invariant which has received considerable attention in number theory, quadratic forms, real algebra and real geometry.

The study of psd elements and sums of squares has a long and rich history. For further reading we refer to Pfister's book on Quadratic Forms [Pf], to Bochnak, Coste and Roy's book on Real Algebraic Geometry BCR, and to the important paper CDLR by Choi, Dai, Lam and Reznick, which contains a wealth of information and ideas. One of the main results of this latter paper was that $p(A)=\infty$ holds whenever $A$ has a real prime ideal $\mathfrak{p}$ for which the local ring $A_{\mathfrak{p}}$ is regular of dimension $\geq 3$ (loc. cit., Thm. 6.6). Moreover, $\mathcal{P}(A) \neq \Sigma(A)$ holds under the same conditions on $A$ ([Sch1, Cor. 1.3]). For example, this applies if $A$ is a finitely generated integral $k$-algebra of dimension $\geq 3$ (where $k$ is a field) whose quotient field $\operatorname{Quot}(A)$ is real.

The proofs of these facts all use, in one way or another, the associated graded ring of a regular local ring, which is a polynomial ring. If the local ring is singular,

Received by the editors November 5, 2002.

2000 Mathematics Subject Classification. Primary 14P99; Secondary 11E25, 32B10, 32 S05.

All authors were supported by the European Research Training Network RAAG (HPRN-CT2001-00271). The first and second named authors were also supported by the Spanish Research Project GAAR (BFM-2002-04797). 
the situation becomes much more complicated in general. A case which has been successfully studied is the class of local analytic rings $A$ of real dimension $\geq 3$ (see below for the notion of real dimension). Here $\mathcal{P}(A) \neq \Sigma(A)$ and $p(A)=\infty$ have been shown in general $\mathrm{Fe} 4$.

These results suggest a negative answer for both the qualitative and the quantitative problem, if the ring in question has dimension at least three. In fact, this is essentially true, and is the content of our main result here. However, the notion of dimension has to be replaced by the real dimension, whose definition we are going to recall.

The real spectrum $\operatorname{Spec}_{r}(A)$ of $A$ consists of all pairs $\alpha=(\mathfrak{p}, \omega)$ where $\mathfrak{p}$ is a prime ideal of $A$ and $\omega$ is an ordering of the residue field $\kappa(\mathfrak{p})$ of $\mathfrak{p}$. The prime ideal $\mathfrak{p}=: \operatorname{supp}(\alpha)$ is called the support of $\alpha$. Alternatively, $\alpha$ can be defined through a homomorphism $\varphi: A \rightarrow k$ into an ordered field; then $\mathfrak{p}=\operatorname{ker}(\varphi)$, and $\omega$ is the restriction to $\kappa(\mathfrak{p})$ of the ordering of $k$. For $f \in A$ one writes $f(\alpha)>0$ (resp. $f(\alpha) \geq 0$, etc.) if the residue class $\bar{f}$ of $f$ in $\kappa(\mathfrak{p})$ is $>0$ (resp. $\geq 0$, etc.) with respect to $\omega$. Thus we can see $f$ as a function on $\operatorname{Spec}_{r}(A)$, and study its sign changes. In particular, matching our previous definition, $f$ is a psd element if and only if $f(\alpha) \geq 0$ for all $\alpha \in \operatorname{Spec}_{r}(A)$. Given a second prime cone $\beta \in \operatorname{Spec}_{r}(A)$, we say that $\alpha$ is a specialization of $\beta$ (written $\beta \rightarrow \alpha$ ) if $f(\alpha)>0$ implies $f(\beta)>0$ for any $f \in A$. This is easily seen to imply $\mathfrak{q}:=\operatorname{supp}(\beta) \subset \operatorname{supp}(\alpha)=\mathfrak{p}$. We put $\operatorname{dim}(\beta \rightarrow \alpha):=\operatorname{dim}\left(A_{\mathfrak{p}} / \mathfrak{q} A_{\mathfrak{p}}\right)$, and define the real dimension of $A$ as

$$
\operatorname{dim}_{r}(A):=\sup \left\{\operatorname{dim}(\beta \rightarrow \alpha): \alpha, \beta \in \operatorname{Spec}_{r}(A), \beta \rightarrow \alpha\right\} .
$$

Therefore, $\operatorname{dim}_{r}(A) \leq \operatorname{dim}(A)$. Equality holds, for example, if $A$ is a domain with real quotient field which is either a finitely generated $k$-algebra or a local analytic ring.

The following is our main theorem. It encompasses the known results on rings of dimension $\geq 3$ mentioned above:

Main Theorem 1.1. Let $A$ be an excellent ring of real dimension at least three. Then $\mathcal{P}(A) \neq \Sigma(A)$ and $p(A)=\infty$.

We enter here the class of excellent rings, which is widely considered as the suitable general setting to work in. In fact, this class includes all interesting rings in algebra and geometry, while being stable under all standard operations.

In Section 2 we will reduce our Main Theorem to the case of complete local domains which are real reduced. Here we say that a ring $A$ is real reduced if $a_{1}^{2}+\cdots+a_{r}^{2}=0$ (with $a_{i} \in A$ ) implies that each $a_{i}=0$. The reduction step is formulated as follows:

Theorem 1.2. Let $k$ be a real field and $A=k\left[\left[x_{1}, \ldots, x_{N}\right]\right]$, where $N$ is a positive integer. Let $\mathfrak{Q}$ be a non-zero prime ideal of $A$ such that the ring $A / \mathfrak{Q}$ is real reduced of dimension $d \geq 3$. Let $k_{0} \subset k$ be a subfield over which $k$ is algebraic.

(i) There exists a polynomial $M \in k_{0}\left[x_{1}, \ldots, x_{d+1}\right]$ with $M(0)=0$ which is psd in $k_{0}\left[x_{1}, \ldots, x_{d+1}\right]$ and is not contained in the $\mathfrak{m}$-adic closure of the set $\Sigma(A)+\mathfrak{Q}$ (where $\mathfrak{m}$ is the maximal ideal of $A$ ).

(ii) For every integer $p \geq 1$ there exists a polynomial $N_{p} \in k_{0}\left[x_{1}, \ldots, x_{d+1}\right]$ which is a sum of $p$ squares of polynomials, but not a sum of $p-1$ squares in the $\operatorname{ring} A / \mathfrak{Q}$.

Note that the hypotheses imply $N>d$, and so $k\left[x_{1}, \ldots, x_{d+1}\right]$ is naturally contained in $A$. That $M$ does not belong to the $\mathfrak{m}$-adic closure of $\Sigma(A)+\mathfrak{Q}$ means 
that there exists an integer $\omega_{0} \geq 0$ such that (the residue class of) $M+f$ is not a sum of squares in $A / \mathfrak{Q}$ for any power series $f \in \mathfrak{m}^{\omega_{0}}$.

Thus, in order to prove the Main Theorem 1.1] it suffices to construct the key polynomials $M, N_{p}$ of 1.2 . This will be achieved in several steps:

1. First, we use local parametrization to replace $\mathfrak{Q}$ by a principal ideal. The geometric idea here is that every complete domain has a birational model which is a hypersurface. However, the use of such a birational model carries spurious denominators which must be controlled. This control is possible because there is a universal denominator, and it is enough to keep track of it. (See (2.1) and Lemma 2.2.)

2. Second, we perform sequences of blowings-up of points and lines over a suitable real closure of $k_{0}$ to uniformize the data and achieve a regular situation. This amounts to desingularizing the hypersurface found in the preceding step. In fact, we do not need desingularization in full, but only local uniformization with a suitable description of strict transforms. The necessary formalism is set up in Section 3, and the regularization is completed in Section 4.

3. Finally, we must address the difficulty that the regularization process involves a ground field extension, so that the polynomials obtained are defined over a finite field extension of $k_{0}$. In order to bring those polynomials down to $k_{0}$, we take norms over $k_{0}$ and use an extra blowing-up of a point. This part is developed in Section 5.

Summing up, from a given domain, we get a birational hypersurface and then a uniformization, to finally come back to the initial domain. This round trip is only possible by a highly delicate control of all of the many data involved. The main technical bulk of it is contained in Sections 4 (especially Proposition 4.4) and 5. Needless to say, we use general ingredients like Cohen's structure theorem or Weierstraß' preparation theorem. Some of these techniques and constructions were already used before, in a less technical setting, by the first-named author [Fe4].

The polynomial $M$ will be a variant of the celebrated Motzkin polynomial. (In fact, any psd polynomial with rational coefficients which is not a sum of squares of real polynomials would do.) The polynomials $N_{p}$ are variants of an ingenious construction by Choi, Dai, Lam and Reznick in CDLR, by which the authors produced, for each $p \geq 1$, a polynomial in $\mathbb{R}[x, y]$ (or in $\mathbb{Z}[x]$ ) which is a sum of $p$, but not of $p-1$, squares.

To end this introduction, we give a brief (incomplete) outline of what is known about the qualitative and the quantitative question for rings of real dimension $\leq 2$. Most known results concern rings of geometric significance. Generally, the answers depend strongly on the particular structure of the ring. This makes the situation quite distinct from the case of dimension $\geq 3$.

Let us first consider the algebraic setting, and let us restrict ourselves to coordinate rings $A=\mathbb{R}[V]$ of affine real algebraic varieties $V$. For curves, we know when $\mathcal{P}=\Sigma$ holds. This property depends on the real singularities of the curve and on its points at infinity ([Sch1], Sch3]). For surfaces, it has been proved that $\mathcal{P}=\Sigma$ if $V$ is non-singular and the set $V(\mathbb{R})$ of real points is compact [Sch4]. As for the Pythagoras number, it is known to be finite (but can be arbitrarily large) for curves [CDLR]. The only Pythagoras number of an algebraic surface $V$ (with 
$V(\mathbb{R})$ Zariski-dense in $V$ ) that seems to have been computed so far is that of the affine plane, which is infinite [CDLR].

Coming to general local rings, $\mathcal{P}=\Sigma$ has been proved for any regular (semi-) local ring $A$ of dimension two Sch2. Moreover, the Pythagoras number $p(A)$ is estimated in terms of the Pythagoras number of the quotient field $K=\operatorname{Quot}(A)$; in particular, $p(A)<4 p(K)$ holds if $p(K)<\infty$ (loc. cit.).

In the local analytic setting, the picture is somewhat more complete. In this case we deal with the ring $\mathcal{O}(X)$ of germs of analytic functions on a real analytic germ $X$. The property $\mathcal{P}=\Sigma$ holds very rarely. Indeed, for each $n \geq 1$, the germ $X=\left\{x_{i} x_{j}=0,1 \leq i<j \leq n\right\}$ is the unique curve germ of embedding dimension $n$ with this property [Sch2], and the embedded surface germs with $\mathcal{P}=\Sigma$ form a small list of multiplicity two germs ([Rz2], [Fe1], FeRz1]). There are a few additional examples of arbitrary multiplicity in higher embedding dimension [Fe3], but the affair seems very mysterious there. As for the Pythagoras number, it is bounded by the multiplicity for curve germs ( $\mathrm{Or}, \mathrm{CaRz}, \mathrm{Qz})$, and by a function of the multiplicity and the embedding dimension for surface germs [Fe2]. Curiously enough, the list of embedded surface germs with $\mathcal{P}=\Sigma$ is also the list of embedded surface germs with Pythagoras number 2 (the minimal number possible) ( $[\mathrm{Rz} 2],[\mathrm{Fe} 3]$ ). On the other hand, the Pythagoras number of a surface germ is the maximum of the Pythagoras numbers of the curve germs it contains FeRz2. Let us also mention that curve germs with high Pythagoras number are ubiquitous: every semianalytic set germ of dimension $\geq 3$ contains (punctured) curve germs with arbitrarily large Pythagoras number (loc. cit.).

There are a variety of other rings (of 'mixed type') for which the Pythagoras number has been computed in [CDLR, like $k[x][[y]]$ or $k[[x]][y]$.

\section{Reduction to the COMplete CASE}

The purpose of this section is to show how our Main Theorem 1.1 can be reduced to the case of complete local domains formulated in 1.2 . Before going into further details we need some notation and terminology related to local parametrization of complete rings. In what follows, $k$ and $K$ will always denote fields of characteristic 0 . As is well known, a complete noetherian local $\operatorname{ring} A$ with residue field $K$ satisfies $A \cong K[[x]] / I$, where $I$ is an ideal of the $\operatorname{ring} K[[x]]$ of formal power series in the indeterminates $x=\left(x_{1}, \ldots, x_{n}\right)$ (to avoid trivial cases we will assume $n \geq 2$ in what follows). If $F \in K[[x]]$ we can write $F=\sum_{d=0}^{\infty} F_{d}$, where $F_{d} \in K[x]$ is a homogeneous polynomial of degree $d$; we denote the order of $F$ by $\omega(F)=$ $\min \left\{d: F_{d} \neq 0\right\}$ (or $\infty$ if $F=0$ ) and the initial form of $F$ by $\operatorname{In}(F)=F_{\omega(F)}$ (or 0 if $F=0)$. We recall that a power series $F \in K[[x]]$ is said to be regular with respect to the variable $x_{i}$ if the power series $g_{i}:=F\left(0, \ldots, 0, x_{i}, 0, \ldots, 0\right) \in K\left[\left[x_{i}\right]\right]$ is not identically zero. We will say that $F$ is totally regular with respect to $x_{i}$ if in addition $\omega\left(g_{i}\right)=\omega(F)$. We have:

2.1. General local parametrization. Let $\mathfrak{q} \subset K[[x]]=K\left[\left[x_{1}, \ldots, x_{n}\right]\right]$ be a nonzero prime ideal and $d=\operatorname{dim}(K[[x]] / \mathfrak{q})$. We say that $\mathfrak{q}$ is properly immersed if for all $d<i \leq n$ there is a monic irreducible polynomial $f_{i} \in \mathfrak{q} \cap K\left[\left[x_{1}, \ldots, x_{i-1}\right]\right]\left[x_{i}\right]$ with $\operatorname{deg}_{x_{i}}\left(f_{i}\right)=\omega\left(f_{i}\right)$ and $\left(f_{d+1}\right)=\mathfrak{q} \cap K\left[\left[x_{1}, \ldots, x_{d}\right]\right]\left[x_{d+1}\right]$. Thus,

$$
\mathfrak{q} \cap K\left[\left[x_{1}, \ldots, x_{d}\right]\right]=(0),
$$


and the inclusion $B_{0}=K\left[\left[x_{1}, \ldots, x_{d}\right]\right] \subset K[[x]] / \mathfrak{q}=B$ is finite. Local parametrization [JP, 3.3.30] says that for any prime ideal $\mathfrak{q} \neq(0)$ there exists a unipotent triangular linear change of the type1

$$
\left(x_{1}+a_{12} x_{2}+\cdots+a_{1 n} x_{n}, x_{2}+a_{23} x_{3}+\cdots+a_{2 n} x_{n}, \ldots, x_{n}\right)
$$

with coefficients $a_{i j}$ in $\mathbb{Z}$ which makes $\mathfrak{q}$ properly immersed.

Assume now that $\mathfrak{q}$ is properly immersed. Let $f=f_{d+1}, p=\operatorname{deg}_{x_{d+1}}(f)=\omega(f)$, and let $\Delta \in K\left[\left[x_{1}, \ldots, x_{d}\right]\right]$ be the discriminant of $f$, all taken with respect to the variable $x_{d+1}$. Then $\theta_{d+1}:=x_{d+1}+\mathfrak{q}$ is a primitive element of $\operatorname{Quot}(B)$ over $\operatorname{Quot}\left(B_{0}\right)$, and

$$
\Delta \cdot B \subset B_{0}+B_{0} \theta_{d+1}+\cdots+B_{0} \theta_{d+1}^{p-1} \cong B^{\prime},
$$

where $B^{\prime}=K\left[\left[x_{1}, \ldots, x_{d+1}\right]\right] /(f)$ (for more details see [JP 1.5.19]). Moreover, we have the following:

Lemma 2.2. Let $\mathfrak{q} \subset K[[x]]$ be a properly immersed ideal and let $d$, the $f_{i}$ and $\Delta$ be as above. Then for any $g \in K[[x]]$ there exists a polynomial

$$
g^{\prime} \in K\left[\left[x_{1}, \ldots, x_{d}\right]\right]\left[x_{d+1}\right],
$$

with $\operatorname{deg}_{x_{d+1}}\left(g^{\prime}\right) \leq p-1$ and $\omega\left(g^{\prime}\right) \geq \omega(g)-\sum_{i=d+1}^{n}\left(\operatorname{deg}\left(f_{i}\right)-1\right)$, such that $g^{\prime}$ is equivalent to $\Delta g$ modulo $\mathfrak{q}$.

Proof. Indeed, $g$ is equivalent modulo $\mathfrak{q}$ to a polynomial $\widehat{g}$ in $x_{d+1}, \ldots, x_{n}$ of order $\geq \omega(g)$ of the type

$$
\widehat{g}=\sum_{\substack{0 \leq \nu_{i} \leq \lambda_{i} \\ d+1 \leq i \leq n}} g_{\nu}\left(x_{1}, \ldots, x_{d}\right) x_{d+1}^{\nu_{d+1}} \cdots x_{n}^{\nu_{n}},
$$

where $g_{\nu} \in K\left[\left[x_{1}, \ldots, x_{d}\right]\right], \nu=\left(\nu_{d+1}, \ldots, \nu_{n}\right)$ and $\lambda_{i}=\operatorname{deg}\left(f_{i}\right)-1$. To see this, just divide $g$ successively by $f_{n}, \ldots, f_{d+1}$ and apply [JP, 3.3.31] to obtain the condition about the order. Then $\omega\left(g_{\nu}\right) \geq \omega(g)-\sum_{i=d+1}^{n} \nu_{i}$,

$$
\Delta g \equiv \Delta \widehat{g} \equiv \sum_{\nu_{i} \leq \lambda_{i}} g_{\nu}\left(x_{1}, \ldots, x_{d}\right)\left(\Delta x_{d+1}^{\nu_{d+1}} \cdots x_{n}^{\nu_{n}}\right) \quad \bmod \mathfrak{q},
$$

and by $(*)$ there exist polynomials $P_{\nu} \in K\left[\left[x_{1}, \ldots, x_{d}\right]\right]\left[x_{d+1}\right]$ of degree $\leq p-1$ such that $\Delta x_{d+1}^{\nu_{d+1}} \cdots x_{n}^{\nu_{n}} \equiv P_{\nu} \bmod \mathfrak{q}$. Thus, $\Delta g \equiv g^{\prime} \bmod \mathfrak{q}$, where $g^{\prime}=$ $\sum_{\nu_{i} \leq \lambda_{i}} g_{\nu}\left(x_{1}, \ldots, x_{d}\right) P_{\nu} \in K\left[\left[x_{1}, \ldots, x_{d}\right]\right]\left[x_{d+1}\right]$ is a polynomial in $x_{d+1}$ of degree $\leq p-1$. Finally, choose a multi-index $\nu^{0}=\left(\nu_{d+1}^{0}, \ldots, \nu_{n}^{0}\right)$ with $\nu_{i}^{0} \leq \lambda_{i}$ $(i=d+1, \ldots, n)$ such that $\min _{\nu}\left\{\omega\left(g_{\nu}\right)\right\}=\omega\left(g_{\nu^{0}}\right)$; then

$$
\begin{aligned}
\omega\left(g^{\prime}\right) & \geq \min _{\nu_{i} \leq \lambda_{i}}\left\{\omega\left(g_{\nu} P_{\nu}\right)\right\} \geq \min _{\nu_{i} \leq \lambda_{i}}\left\{\omega\left(g_{\nu}\right)\right\}=\omega\left(g_{\nu^{0}}\right) \\
& \geq \omega(g)-\sum_{i=d+1}^{n} \nu_{i}^{0} \geq \omega(g)-\sum_{i=d+1}^{n} \lambda_{i} .
\end{aligned}
$$

Now, we proceed with the announced reduction.

\footnotetext{
${ }^{1}$ The coefficients of the change of coordinates can be taken over $\mathbb{Z}$ because there is no non-zero polynomial in $n$ variables which vanishes on $\mathbb{Z}^{n}$; for more details see 4.2
} 
2.3. Reduction of Theorem 1.1 to Theorem 1.2. Since the real dimension of $A$ is $\geq 3$, there exists a specialization $\beta \rightarrow \alpha$ of dimension $d \geq 3$ in $\operatorname{Spec}_{r}(A)$. If we write $\mathfrak{q}=\operatorname{supp}(\beta)$ and $\mathfrak{p}=\operatorname{supp}(\alpha)$, then the excellent local domain $B=A_{\mathfrak{p}} / \mathfrak{q} A_{\mathfrak{p}}$ has dimension $d \geq 3$. Let $k$ be the residue field and $\mathfrak{m}$ the maximal ideal of $B$. Then $\alpha$ induces an ordering on $k \cong A_{\mathfrak{p}} / \mathfrak{p} A_{\mathfrak{p}} \cong \operatorname{Quot}(A / \mathfrak{p})$, and $\beta$ induces an ordering in $B$ with support (0). Thus, $B$ is a real reduced local domain of dimension $d \geq 3$ with real residue field. Moreover, there exists a quasi-coefficient field $k_{0}$ in $A_{\mathfrak{p}}$, i.e, $k_{0}$ is a maximal subfield of $A_{\mathfrak{p}}$ such that $k$ is algebraic over $k_{0}$ [Mt, 38.E-F]. Note that $k_{0}$ is a subfield of $B$.

Let $\widehat{B}$ be the $\mathfrak{m}$-adic completion of $B$ and $\widehat{\mathfrak{m}}$ the maximal ideal of $\widehat{B}$. By $\mathrm{ABR}$ VII.3.2], there exists a specialization $\widehat{\beta} \rightarrow \widehat{\alpha}$ in $\operatorname{Spec}_{r}(\widehat{B})$ lying over $\beta \rightarrow \alpha$. Therefore $\operatorname{supp}(\widehat{\alpha})=\widehat{\mathfrak{m}}$, and $\widehat{\mathfrak{q}}:=\operatorname{supp}(\widehat{\beta})$ is a minimal prime ideal of $\widehat{B}$. Thus, since $B$ is excellent, $\operatorname{dim} B=\operatorname{dim}(\widehat{B} / \widehat{\mathfrak{q}})$. On the other hand, since $A$ is noetherian, there exist $a_{1}, \ldots, a_{N} \in \mathfrak{p}$ which generate $\mathfrak{p}$; therefore, $\overline{a_{1}}, \ldots, \overline{a_{N}}$ generate the maximal ideal of $B$ (where $\overline{a_{i}}:=a_{i}+\mathfrak{q} A_{\mathfrak{p}}$ ). Since $k$ has characteristic 0 , we deduce, by the structure theorem of complete local rings [Ng, V.31.1], that $\widehat{B} \supset k$ and the homomorphism $k\left[\left[x_{1}, \ldots, x_{N}\right]\right] \rightarrow \widehat{B}, x_{i} \mapsto \overline{a_{i}}$ is surjective. Thus, if $I$ is its kernel, we have $\widehat{B} \cong k\left[\left[x_{1}, \ldots, x_{N}\right]\right] / I$. Let $\mathfrak{Q}$ be the prime ideal of $k\left[\left[x_{1}, \ldots, x_{N}\right]\right]$ such that $\mathfrak{Q} / I$ corresponds to $\widehat{\mathfrak{q}}$. We can assume $\mathfrak{Q} \neq(0)$ by adding an extra variable $x_{N+1}$ if necessary. Therefore $\operatorname{dim} \widehat{B}=d<N$. By (2.1) we can suppose that $\mathfrak{Q}$ is properly immersed Let $n:=d+1$ and $x=\left(x_{1}, \ldots, x_{n}\right)$ (note that $\left.4 \leq n \leq N\right)$. We have the following commuting diagram (where we write $\underline{x}:=\left(x_{1}, \ldots, x_{N}\right)$ ):

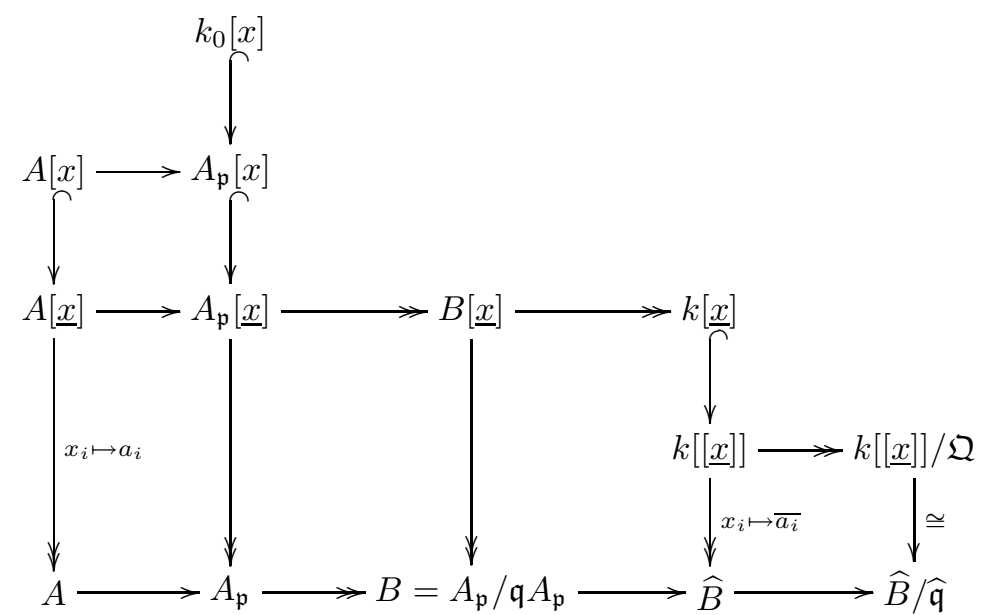

In the bottom row, the last three rings have the same dimension $d$; moreover, $k[[\underline{x}]] / \mathfrak{Q}=k\left[\left[x_{1}, \ldots, x_{N}\right]\right] / \mathfrak{Q}$ is a real reduced domain. Now, there exist polynomials $M, N_{p} \in k_{0}[x](p \geq 1)$ satisfying conditions (i) and (ii) of Theorem 1.2. We recall that condition (i) says that $M$ is psd in $k_{0}[x]$ with $M(0)=0$, and that there exists an integer $\omega_{0} \geq 0$ such that $M+f$ is not a sum of squares in $k\left[\left[x_{1}, \ldots, x_{N}\right]\right] / \mathfrak{Q}$ for any $f \in \mathfrak{m}^{\omega_{0}}$.

\footnotetext{
${ }^{2}$ One of the advantages of a unipotent triangular linear change of coordinates with coefficients in $\mathbb{Z}$ is that we keep in $A$ the generators of the maximal ideal of $\widehat{B}$. We will need later, almost at the end of the article, to have $\mathfrak{Q}$ properly immersed, because this allows us to use 2.2 .
} 
Using this, we prove first that $p(A)=\infty$. Recall that $k_{0}$ is a maximal subfield of $A_{\mathfrak{p}}$. Write $N_{p}=N_{1 p}^{2}+\cdots+N_{p p}^{2}$ with $N_{i p} \in k_{0}[x]$. Choose $c_{p} \in A \backslash \mathfrak{p}$ so that $c_{p} N_{i p}$ has coefficients in $A$ for $i=1, \ldots, p$, and let

$$
S_{p}:=\sum_{i=1}^{p} c_{p}^{2} N_{i p}\left(a_{1}, \ldots, a_{n}\right)^{2} \in A .
$$

Then $S_{p}$ is a sum of $p$ squares in $A$. From the diagram and the conditions on $N_{p}$ above we deduce that $S_{p}$ is not a sum of $p-1$ squares in $A$. Since this can be done for all $p$, we conclude that $p(A)=\infty$.

Second, in order to prove $\mathcal{P}(A) \neq \Sigma(A)$, we construct from $M$ an element $R \in \mathcal{P}(A) \backslash \Sigma(A)$. Choose $b \in A \backslash \mathfrak{p}$ such that $b M$ has coefficients in $A$, and put $P:=b^{2} M\left(a_{1}, \ldots, a_{n}\right) \in A$. Clearly, since $M(0)=0$, we have $P \in \mathfrak{p}$. Let $\gamma \in \operatorname{Spec}_{r}(A)$ be a prime cone. First, if $\operatorname{supp}(\gamma) \subset \mathfrak{p}$, we can see $\gamma$ as an element of $\operatorname{Spec}_{r}\left(A_{\mathfrak{p}}\right)=\left\{\eta \in \operatorname{Spec}_{r}(A): \operatorname{supp}(\eta) \subset \mathfrak{p}\right\}$. Since the map

$$
\begin{aligned}
k_{0}[x] & \rightarrow A_{\mathfrak{p}}, \\
G & \mapsto G\left(a_{1}, \ldots, a_{n}\right),
\end{aligned}
$$

is a homomorphism and $b^{2} M$ is psd in $k_{0}[x]$, we conclude that $P(\gamma) \geq 0$. On the other hand, if $\operatorname{supp}(\gamma) \supset \mathfrak{p}$, then we have $P(\gamma)=0$.

Now consider the open subset $U=\{P<0\}$ of $\operatorname{Spec}_{r}(A)$. For each $\gamma \in U$ we have $\mathfrak{p} \not \subset \operatorname{supp}(\gamma) \not \subset \mathfrak{p}$; hence, $\left(a_{1}^{2}+\cdots+a_{N}^{2}\right)(\gamma)>0$ (because if $a_{1}^{2}+\cdots+a_{N}^{2} \in \operatorname{supp}(\gamma)$ then $\mathfrak{p}=\left(a_{1}, \ldots, a_{N}\right) \subset \operatorname{supp}(\gamma)$, impossible). Pick $g_{\gamma} \in \operatorname{supp}(\gamma) \backslash \mathfrak{p}$. Then we have

$$
\left(g_{\gamma}^{2} P+\left(a_{1}^{2}+\cdots+a_{N}^{2}\right)^{\omega_{0}}\right)(\gamma)=\left(a_{1}^{2}+\cdots+a_{N}^{2}\right)^{\omega_{0}}(\gamma)>0
$$

(where $\omega_{0}$ is as above). Therefore the open constructible sets

$$
U_{\gamma}=\left\{g_{\gamma}^{2} P+\left(a_{1}^{2}+\cdots+a_{N}^{2}\right)^{\omega_{0}}>0\right\} \subset \operatorname{Spec}_{r}(A) \quad(\gamma \in U)
$$

cover $U$. Since $U$ is quasi-compact [BCR 7.1.13], there exist $U_{\gamma_{1}}, \ldots, U_{\gamma_{t}}$ covering $U$. Therefore $\operatorname{Spec}_{r}(A)=\{P \geq 0\} \cup U_{\gamma_{1}} \cup \cdots \cup U_{\gamma_{t}}$. Put $g_{j}:=g_{\gamma_{j}}$ and consider

$$
R:=\left(\prod_{j=1}^{t} g_{j}^{2}\right) \cdot P+\left(\sum_{j=1}^{t} \prod_{i \neq j} g_{i}^{2}\right) \cdot\left(a_{1}^{2}+\cdots+a_{N}^{2}\right)^{\omega_{0}} .
$$

We claim that $R \in \mathcal{P}(A) \backslash \Sigma(A)$. Let $\gamma \in \operatorname{Spec}_{r}(A)$. If $\gamma \notin U$, then it is clear that $R(\gamma) \geq 0$, so we can suppose that $\gamma \in U$. Then $\gamma \in U_{\gamma_{\ell}}$ for some $\ell$, and we have

$$
R=\left(\prod_{j \neq \ell} g_{j}^{2}\right) \cdot\left(g_{\ell}^{2} P+\left(a_{1}^{2}+\cdots+a_{N}^{2}\right)^{\omega_{0}}\right)+\left(\sum_{j \neq \ell} \prod_{i \neq j} g_{i}^{2}\right) \cdot\left(a_{1}^{2}+\cdots+a_{N}^{2}\right)^{\omega_{0}},
$$

which is clearly $\geq 0$ at $\gamma$. If $R \in \Sigma(A)$, then, since $g_{j} \notin \mathfrak{p}$, we will conclude from the diagram above that there exists $\vartheta \in k$ such that $M(x)+\vartheta\left(x_{1}^{2}+\cdots+x_{N}^{2}\right)^{\omega_{0}}$ is a sum of squares in $k\left[\left[x_{1}, \ldots, x_{N}\right]\right] / \mathfrak{Q}$, against our construction.

\section{TRANSFORMS}

The purpose of this section is to settle all the notation and terminology about desingularization and strict transforms that we will need along the way. In what follows we set $x=\left(x_{1}, \ldots, x_{n}\right)$, and $K$ will denote a field of characteristic 0 . 
3.1. Strict transforms. Given a field $K$, we will mainly use homomorphisms $\varphi^{*}$ : $K[[x]] \rightarrow K[[x]]$ induced by transforms $\varphi: K^{n} \rightarrow K^{n}$ of the following types:

(a) linear changes: $\varphi(x)=A x, A \in \mathrm{GL}_{n}(K)$,

(b) local blowings-up of points: $\varphi(x)=\left(x_{1}, x_{1} x_{2}, \ldots, x_{1} x_{n}\right)$,

(c) local blowings-up of lines: $\varphi(x)=\left(x_{1}, x_{2}, x_{1} x_{3}, \ldots, x_{1} x_{n}\right)$.

A finite sequence of transforms $\varphi_{1}, \ldots, \varphi_{r}$ will be denoted by $T=\left[\varphi_{1}, \ldots, \varphi_{r}\right]$. For a series $f \in K[[x]]$ we will denote $f \odot \varphi=\varphi^{*}(f)=f(\varphi(x))$. Note that if $f \in K[x]$ is a polynomial, up to identification with the associated polynomial function, we have $f \odot \varphi=f \circ \varphi$.

More generally, if $h_{1}, \ldots, h_{n} \in K[[x]]$ with $h_{i}(0)=0$ and $h=\left(h_{1}, \ldots, h_{n}\right)$, we can also consider the homomorphism $h^{*}: K[[x]] \rightarrow K[[x]], x_{i} \mapsto h_{i}$. For any series $f \in K[[x]]$ we write $f \odot h=h^{*}(f)=f\left(h_{1}, \ldots, h_{n}\right)$.

Let $m$ be a positive integer. For any $f=\left(f_{1}, \ldots, f_{m}\right) \in K[[x]]^{m}$ and any sequence $T=\left[\varphi_{1}, \ldots, \varphi_{r}\right]$ (the $\varphi_{i}$ 's as above) we will denote $f \odot T=f \odot \varphi_{1} \odot \cdots \odot \varphi_{r}$. We define the strict transform $\widetilde{f \odot T}$ of $f$ via $T$ inductively. First, if $T=\left[\varphi_{1}\right]$ and

(a) $\varphi_{1}$ is a linear change: $\widetilde{f \odot T}:=f \odot \varphi_{1}$,

(b) $\varphi_{1}$ is as in (b) or (c): $\widetilde{f \odot T}:=\left(f \odot \varphi_{1}\right) / x_{1}^{\mu}$, where $\mu$ is the greatest integer such that $x_{1}^{\mu}$ divides $f_{i} \odot \varphi_{1}$ for all $i$.

Next, if $T:=\left[\varphi_{1}, \ldots, \varphi_{r}\right]$ with $r \geq 2$ we define $\widetilde{f \odot T}$ as the strict transform of $\widetilde{f \odot \varphi_{1}}$ via $\left[\varphi_{2}, \ldots, \varphi_{r}\right]$.

Notice that the strict transform of a tuple (via a finite sequence of transforms) is not, in general, the tuple of the strict transforms of the components of the tuple.

If $f \in K[[x]]$ is a series and $\varphi$ a blowing-up of a point, then $f \odot \varphi=x_{1}^{\omega(f)}(\widetilde{f \odot \varphi)}$ and $\omega(\widetilde{f \odot \varphi}) \leq \omega(f)$. Moreover, we have the following result whose proof, although well known, is included here for the sake of the reader:

Lemma 3.2. Let $r \geq 2$ and $A=K[x]$ or $K[[x]]$. Let $f, f_{1}, \ldots, f_{r} \in A$ and $T a$ sequence of transforms. Then:

(i) if $f$ has no multiple factors in $A$, then neither has $\widetilde{f \odot T}$;

(ii) if $f_{1}, \ldots, f_{r}$ are relatively prime in $A$, then so are $\widetilde{f_{1} \odot T}, \ldots, \widetilde{f_{r} \odot T}$.

We will only prove 3.2 for $A=K[[x]]$, the case $A=K[x]$ being similar (and, in fact, easier).

Proof of Lemma 3.2. First, we recall that an element $a$ of a domain $A$ is reduced if the ideal ( $a$ ) is radical; if $A$ is a UFD, this happens if and only if $a$ has no multiple factors.

It is enough to prove that if $1 \leq m \leq 2$ and $\varphi(y)=\left(y_{1}, \ldots, y_{m}, y_{1} y_{m+1}, \ldots, y_{1} y_{n}\right)$, then (i) and (ii) hold for $T=[\varphi]$. This local blowing-up can be described as follows. Write $y_{i}=x_{i}$ if $1 \leq i \leq m$, and $y_{i}=x_{i} / x_{1}$ if $m+1 \leq i \leq n$, and let $A^{\prime}=A\left[y_{r+1}, \ldots, y_{n}\right]_{\left(y_{1}, \ldots, y_{n}\right)}$, considered as a subring of $\operatorname{Quot}(A)=K((x))$. Let $\widehat{A^{\prime}}$ be the completion of $A^{\prime}$ with respect to the $\left(y_{1}, \ldots, y_{n}\right)$-adic topology. Then $\varphi^{*}$ is the composition

$$
\varphi^{*}: A=K[[x]] \subset A^{\prime} \subset \widehat{A^{\prime}} \cong K[[y]]=K\left[\left[y_{1}, \ldots, y_{n}\right]\right] .
$$

We have $\widehat{A^{\prime}} \cong K[[y]]$, since $A^{\prime}$ is a regular local ring with regular system of parameters $y_{1}, \ldots, y_{n}$ and residue field $K$. Clearly, $A^{\prime}\left[1 / y_{1}\right]$ is a localization of $A\left[1 / y_{1}\right]$; hence an irreducible element of $A$ is either irreducible in $A^{\prime}$, or a unit, or a unit 
times a power of $y_{1}$. Thus, if $f$ is reduced in $A$ it is so in $A^{\prime}$, except maybe for a power of $y_{1}$, which is irrelevant for the strict transform. Finally, since $A^{\prime}$ is excellent, every element reduced in $A^{\prime}$ is also reduced in $\widehat{A^{\prime}}$ [Mt, 33.B, Lemma 2], and we conclude that $\widetilde{f \odot T}$ is also reduced.

Now we consider statement (ii). We first prove it for $r=2$. For this particular case it is enough to check that if $f, g \in K[[x]]$ are non-associated irreducible series, then the series $\widetilde{f \odot T}, \widetilde{g \odot T}$ are relatively prime. Consider the reduced series $f g \in$ $K[[x]]$. From the definition of the strict transform and from (i) it follows that $\widetilde{(f g) \odot T}=\widetilde{f \odot T} \cdot \widetilde{g \odot T}$ is reduced. But this means that the series $\widetilde{f \odot T}, \widetilde{g \odot T}$ are relatively prime.

The general case $r \geq 3$ follows from the case $r=2$, since $(1)(\widetilde{f g) \odot T}=\widetilde{f \odot T}$. $\widetilde{g \odot T}$ for any series $f, g \in A$, and (2) if $f \in A$ is irreducible and $h \in \widehat{A^{\prime}}$ is an irreducible factor of $\widetilde{f \odot T}$ then $\left(\varphi^{*}\right)^{-1}\left(g \widehat{A^{\prime}}\right)=f A$.

Lemma 3.3. Let $T=\left[\varphi_{1}, \ldots, \varphi_{r}\right]$ be a sequence of transforms and $m$ a positive integer. Then there exist finitely many polynomials $q_{1}, \ldots, q_{\ell} \in K[x] \subset K[[x]]$ such that for every $f=\left(f_{1}, \ldots, f_{m}\right) \in K[[x]]^{m}$ we have $f \odot T=q_{1}^{\nu_{1}} \cdots q_{\ell}^{\nu_{\ell}}(\widetilde{f \odot T)}$ for suitable integers $\nu_{1}, \ldots, \nu_{\ell} \geq 0$. Moreover, $\widetilde{f \odot T}=\left(g_{1}, \ldots, g_{m}\right)$ is relatively prime with all $q_{i}$ 's, that $i s, \operatorname{gcd}\left(g_{1}, \ldots, g_{m}, q_{i}\right)=1$ for each $i$.

Proof. We proceed by induction on $r$. For $r=1$ :

(i) if $\varphi_{1}$ is a linear change, then $f \odot T=\widetilde{f \odot T}$, and

(ii) if $\varphi_{1}$ is a local blowing-up then $f \odot T=x_{1}^{\nu_{1}} \widetilde{f \odot T}$, and then $x_{1}$ and $\widetilde{f \odot T}$ are relatively prime.

Now suppose $r>1$, and let $T_{1}=\left[\varphi_{1}, \ldots, \varphi_{r-1}\right]$. By the induction hypothesis there exist finitely many polynomials $p_{1}, \ldots, p_{\ell} \in K[x]$ such that for every $f \in$ $K[[x]]^{m}$

$$
f \odot T_{1}=p_{1}^{\nu_{1}} \cdots p_{\ell}^{\nu_{\ell}}\left(\widetilde{f \odot T_{1}}\right), \quad \nu_{1}, \ldots, \nu_{\ell} \geq 0,
$$

and $\widetilde{f \odot T_{1}}$ is relatively prime with all $p_{i}$. Therefore,

$$
f \odot T=f \odot T_{1} \odot \varphi_{r}=\left(p_{1} \odot \varphi_{r}\right)^{\nu_{1}} \cdots\left(p_{\ell} \odot \varphi_{r}\right)^{\nu_{\ell}}\left(\widetilde{f \odot T_{1}} \odot \varphi_{r}\right) .
$$

Again we distinguish two cases:

(i) If $\varphi_{r}$ is a linear change, we take $q_{i}=p_{i} \odot \varphi_{r} \in K[x]$ for all $i$.

(ii) If $\varphi_{r}$ is a local blowing-up, factoring out all $x_{1}$ 's we get

$$
f \odot T=x_{1}^{\nu_{\ell+1}}\left(\widetilde{p_{1} \odot \varphi_{r}}\right)^{\nu_{1}} \cdots\left(\widetilde{p_{\ell} \odot \varphi_{r}}\right)^{\nu_{\ell}}(\widetilde{f \odot T})
$$

and we take $q_{i}=\widetilde{p_{i} \odot \varphi_{r}} \in K[x]$ for $i=1, \ldots, \ell$ and $q_{\ell+1}=x_{1}$.

It is clear from (ii) above and $\left[3.2\right.$ (ii) that $\widetilde{f \odot T}$ is relatively prime with $q_{i}$ for $i=1, \ldots, \ell$, and with $q_{\ell+1}$ by the definition of the strict transform.

3.4. Reduced polynomial transforms. Let $T=\left[\varphi_{1}, \ldots, \varphi_{r}\right]$ be a sequence of transforms with coefficients in a field $K$. The polynomial map $\varphi_{1} \circ \cdots \circ \varphi_{r}$ : $K^{n} \rightarrow K^{n}$, which is in fact a birational map, induces a $K$-automorphism $\Phi$ of $K(x)$ which leaves $K[x]$ invariant, by $\Phi(f)=f \odot T(f \in K[x])$. Let $\Phi^{-1}$ be the inverse automorphism of $K(x)$. There exist polynomials $0 \neq f_{1}, \ldots, f_{n}, g \in K[x]$ with $\operatorname{gcd}\left(f_{1}, \ldots, f_{n}, g\right)=1$ and $\Phi^{-1}\left(x_{i}\right)=\frac{f_{i}}{g}(i=1, \ldots, n)$. Note that $f_{1}, \ldots, f_{n}, g$ 
are unique up to a common factor in $K^{*}$. Now consider the birational map $T^{-1}$ : $K^{n} \rightarrow K^{n}$ inverse to $\varphi_{1} \circ \cdots \circ \varphi_{r}$ defined by

$$
T^{-1}(x)=\left(\frac{f_{1}(x)}{g(x)}, \ldots, \frac{f_{n}(x)}{g(x)}\right)
$$

for $x \notin\{g=0\}$. In what follows we will identify the tuple $\left(\frac{f_{1}}{g}, \ldots, \frac{f_{n}}{g}\right)$ with the birational map $T^{-1}$. Now, given a homogeneous polynomial $P \in K[x]$ of degree $d$, we define a reduced polynomial transform of $P$ with respect to $T^{-1}$ by

$$
\left(P \odot T^{-1}\right)^{\vee}:=g^{d} \Phi^{-1}(P)=P\left(f_{1}, \ldots, f_{n}\right) \in K[x] .
$$

Note that $\left(P \odot T^{-1}\right)^{\vee}$ is unique up to multiplication by a value in $\left(K^{*}\right)^{d}$.

Remarks 3.5. Let $T$ be a sequence of transforms. By 3.3 there exist finitely many polynomials $q_{1}, \ldots, q_{\ell} \in K[x] \subset K[[x]]$ such that for every $f \in K[[x]]$ we have $f \odot T=q^{\nu}\left(\widetilde{f \odot T)}\right.$ for suitable integers $\nu_{1}, \ldots, \nu_{\ell} \geq 0$, where $q^{\nu}=q_{1}^{\nu_{1}} \cdots q_{\ell}^{\nu_{\ell}}$, and $\widetilde{f \odot T}$ is relatively prime with all $q_{j}$ 's. Let $f_{1}, \ldots, f_{n}, g \in K[x]$ be relatively prime polynomials such that $T^{-1}=\left(\frac{f_{1}}{g}, \ldots, \frac{f_{n}}{g}\right)$. Then $\widetilde{g \odot T}=u \in K^{*}$. Moreover, let us prove that if $P \in K[x]$ is a homogeneous polynomial of degree $d$ with $\operatorname{gcd}\left(P, \prod_{j=1}^{\ell} q_{j}\right)=1$, then the strict transform of $\left(P \odot T^{-1}\right)^{\vee}$ via $T$ is $u^{d} P$.

Indeed, since $\Phi\left(f_{i}\right)=q^{\mu_{i}} \widetilde{f_{i} \odot T}$ and $\Phi(g)=q^{\mu_{0}} \widetilde{g \odot T}$, where $\mu_{i}=\left(\mu_{i 1}, \ldots, \mu_{i \ell}\right)$ and $\mu_{i j} \geq 0$ are integers for $i=0, \ldots, n$ and $j=1, \ldots, \ell$, then

$$
x_{i}=\Phi\left(\frac{f_{i}}{g}\right)=q^{\mu_{i}-\mu_{0}} \frac{\widetilde{f_{i} \odot T}}{\widetilde{g \odot T}} \quad \text { for } i=1, \ldots, n .
$$

By 3.2 (ii) for $A=K[x]$, the polynomials $\widetilde{f_{1} \odot T}, \ldots, \widetilde{f_{n} \odot T}, \widetilde{g \odot T}$ are relatively prime because so are the polynomials $f_{1}, \ldots, f_{n}, g$. Thus, since $\widehat{g \odot T}$ is relatively prime to all the $q_{j}$ 's, we deduce that $\widetilde{g \odot T}$ must be a unit of $K[x]$. Hence, $\widetilde{g \odot T}=u \in K^{*}$.

On the other hand,

$$
P=\Phi \circ \Phi^{-1}(P)=\Phi\left(\frac{\left(P \odot T^{-1}\right)^{\vee}}{g^{d}}\right)=\frac{\Phi\left(\left(P \odot T^{-1}\right)^{\vee}\right)}{q^{\mu_{0}} u^{d}}
$$

and then $\Phi\left(\left(P \odot T^{-1}\right)^{\vee}\right)=(g \odot T)^{d} P=q^{\mu_{0}} u^{d} P$. Hence, since $\operatorname{gcd}\left(P, \prod_{j=1}^{\ell} q_{j}\right)=$ 1 , we conclude that the strict transform of $\left(P \odot T^{-1}\right)^{\vee}$ via $T$ is $u^{d} P$.

\section{LOCAL UNIFORMIZATION OF A HYPERSURFACE}

In this section we prove several technical results about local uniformization of hypersurfaces which will allow us to prove 1.2. Let $k$ always be a field.

Lemma 4.1. Let $x=\left(x_{1}, \ldots, x_{n}\right)$, and let $f \in k[[x]]$ be a series with $f(0)=0$ and without multiple factors and such that the ring $k[[x]] /(f)$ is real reduced. Then, there exists an ordering $\alpha$ of $k$ and a finite sequence $T$ of transforms with coefficients in the real closure $R$ of $(k, \alpha)$, such that the strict transform of $f$ via $T$ in $R[[x]]$ is

$$
\widetilde{f \odot T}=\left(x_{1}-h\left(x_{2}, \ldots, x_{n}\right)\right) U
$$

where $h \in R\left[\left[x_{2}, \ldots, x_{n}\right]\right]$ has order $\geq 2$ and $U \in R[[x]]$ is a unit. 
Proof. After a linear change of coordinates we can suppose that $f$ is regular with respect to $x_{n}$ of order $\omega(f)$. By Weierstraß' Preparation Theorem, there exist a Weierstraß polynomial $P \in k\left[\left[x_{1}, \ldots, x_{n-1}\right]\right]\left[x_{n}\right]$ and a unit $V \in k[[x]]$ such that $f=P V$; hence $k[[x]] /(f)=k[[x]] /(P)$. We claim that there exists a prime cone $\beta \in \operatorname{Spec}_{r}(k[[x]])$ such that $P(\beta)<0$. Otherwise, $P$ would be a sum of squares of meromorphic series. Thus, there would exist relatively prime series $a, a_{1}, \ldots, a_{r} \in$ $k[[x]]$ such that $a^{2} P=a_{1}^{2}+\cdots+a_{r}^{2}$. Since $k[[x]] /(P)$ is a real reduced ring, $P$ divides $a_{1}, \ldots, a_{r}$ and therefore $P^{2}$ divides $a^{2} P$. Thus, $P$ (which is reduced) divides $a$, contradicting $\operatorname{gcd}\left(a, a_{1}, \ldots, a_{r}\right)=1$.

Now, since $k[[x]]$ is a local henselian ring with residue field $k$, there exists an ordering $\alpha$ of $k$ such that $\beta \rightarrow \alpha$ [ABR II.2.4]. Next, by the curve selection lemma ABR, VII.4.1], there exist formal power series $x(t)=\left(x_{1}(t), \ldots, x_{n}(t)\right)$ with coefficients in the real closure $R=\kappa(\alpha)$ of $(k, \alpha)$ and $x_{i}(0)=0$ for all $i$ such that $P(x(t))=c t^{q}+$ higher order terms, with $c<0$. On the other hand, if we substitute $x_{1}=0, \ldots, x_{n-1}=0, x_{n}=t$ into $P$, we get $P(0, t)=t^{\operatorname{deg}_{x_{n}}(P)}$. Hence, $P$ takes both strictly positive and strictly negative values on the real spectrum of $R[[x]]$. Moreover, since $P$ has no multiple factors in $k[[x]]$, the same is true in $R[[x]]$. Finally, proceeding as in the proof of [Fe4, 2.2,2.3] (just replace $\mathbb{R}\{x\}$ by $R[[x]]$ and the ordinary composition $\circ$ by $\odot$ ), we obtain the desired result.

We recall the following fact, whose proof is an easy exercise:

Lemma 4.2. Let $R \subset S$ be an extension of rings such that $S$ is a domain of characteristic zero, and let $f \in S\left[x_{1}, \ldots, x_{m}\right]$ be a nonzero polynomial. Then there exists $c \in R^{m}$ such that $f(c) \neq 0$.

Lemma 4.3. Let $P, Q, R \in K[[x]]$ be nonzero series. Then $\operatorname{gcd}(P+c Q, R)=$ $\operatorname{gcd}(P, Q, R)$ for all $c \in K$ except maybe for finitely many values.

Proof. Let $D=\operatorname{gcd}(P, Q, R)$ and $P_{1}, Q_{1}, R_{1} \in K[[x]]$ such that $P=P_{1} D, Q=$ $Q_{1} D, R=R_{1} D$; then we have that $\operatorname{gcd}\left(P_{1}, Q_{1}, R_{1}\right)=1$. If $\operatorname{gcd}\left(P_{1}+c Q_{1}, R_{1}\right) \neq 1$ for infinitely many $c \in K$, then there exist $c_{1} \neq c_{2}$ in $K$ and an irreducible factor $F \in K[[x]]$ of $R_{1}$ such that $F \mid P_{1}+c_{i} Q_{1}$ for $i=1,2$. Hence, $F \mid P_{1}$ and $F \mid Q_{1}$, a contradiction.

We finish this section with the following key result for 1.2

Proposition 4.4. Let $k$ be a real field and $k_{0}$ a subfield of $k$ over which $k$ is algebraic. Let $x=\left(x_{1}, \ldots, x_{n}\right)$, and let $f \in k[[x]]$ be a non-unit such that the ring $k[[x]] /(f)$ is real reduced. Let $\Delta \in k[[x]]$ be a series relatively prime to $f$. Then, there exist:

- an ordering $\alpha$ of $k$ with real closure $R$,

- a finite Galois extension $L \mid k_{0}(L \subset R[\sqrt{-1}])$,

- a sequence of transforms $T=\left[\varphi_{1}, \ldots, \varphi_{r}\right]$ with coefficients in $L \cap R$, and

- a power series $h \in R\left[\left[x_{2}, \ldots, x_{n}\right]\right]$ with $\omega(h) \geq 2$,

such that, given

$\star$ a field $E \subset L \cap R$ that contains $k_{0}$,

$\star$ finitely many automorphisms $\sigma_{1}, \ldots, \sigma_{s} \in \operatorname{Gal}\left(L \mid k_{0}\right)$ with $\left.\sigma_{i}\right|_{E} \neq \mathrm{id}$, and

$\star\left(\psi_{10}, \psi_{11}, \ldots, \psi_{1 n}\right), \ldots,\left(\psi_{s 0}, \psi_{s 1}, \ldots, \psi_{s n}\right) \in L[[x]]^{n+1}$, 
there exists a linear change $\Gamma_{c}\left(x_{2}, \ldots, x_{n}\right)=\left(x_{2}, x_{3}+c_{3} x_{2}, \ldots, x_{n}+c_{n} x_{2}\right)$, where $c=\left(c_{3}, \ldots, c_{n}\right) \in E^{n-2}$, that has the following properties $\left.A\right)$ and $\left.B\right)$. In what follows we write $\tau\left(x_{2}, \ldots, x_{n}\right):=\left(h, x_{2}, \ldots, x_{n}\right)$ and $\rho\left(x_{2}, \ldots, x_{n}\right):=\left(x_{2}, x_{2} x_{3}, \ldots, x_{2} x_{n}\right)$.

A) Let $g \in k[[x]]$ be such that $g \odot T \odot \tau \neq 0$ and let $\widetilde{g}$ be the strict transform of $\widetilde{g \odot T} \odot \tau$ via $\left[\Gamma_{c}, \rho\right]$. Then:

i) There exist an integer $d_{g} \geq 0$ and a unit $U_{g} \in R\left[\left[x_{2}, \ldots, x_{n}\right]\right]$ such that

$$
g \odot T \odot \tau \odot \Gamma_{c} \odot \rho=x_{2}^{d_{g}} U_{g} \widetilde{g} .
$$

ii) Let

$$
\begin{gathered}
\Gamma_{c}^{\prime}:=\left(x_{1}, \Gamma_{c}\left(x_{2}, \ldots, x_{n}\right)\right), \quad \rho^{\prime}:=\left(x_{1}, \rho\left(x_{2}, \ldots, x_{n}\right)\right), \\
\tau^{\prime}:=\left(h^{\prime}, x_{2}, \ldots, x_{n}\right),
\end{gathered}
$$

where $h^{\prime}=h \odot \Gamma_{c} \odot \rho$ and let $T_{c}^{\prime}=\left[\varphi_{1}, \ldots, \varphi_{r}, \Gamma_{c}^{\prime}, \rho^{\prime}\right]$. Then, there is an integer $\delta_{g} \geq 0$ such that $\widetilde{g \odot T_{c}^{\prime}} \odot \tau^{\prime}=x_{2}^{\delta_{g}} \widetilde{g}$.

iii) There is an integer $\omega_{1} \geq 0$ such that if there exists an equation of the type

$$
\Delta^{2} g+\zeta=h_{1}^{2}+\cdots+h_{p}^{2}+f Q, \quad \text { where } h_{1}, \ldots, h_{p}, Q, \zeta \in k[[x]],
$$

and $\omega(\zeta) \geq \omega_{1}$, then there is a series $\xi \in R\left[\left[x_{2}, \ldots, x_{n}\right]\right]$ with $\omega(\xi)>\omega(\widetilde{g})$ such that either $\widetilde{g}+\xi$ or $-(\widetilde{g}+\xi)$ is a sum of $p$ squares in $R\left[\left[x_{2}, \ldots, x_{n}\right]\right]$. Moreover, if $\zeta=0$ then we can asume $\xi=0$, that is, either $\widetilde{g}$ or $-\widetilde{g}$ is a sum of $p$ squares in $R\left[\left[x_{2}, \ldots, x_{n}\right]\right]$.

B) Let $\Psi_{i}=\left(\psi_{i 1}, \psi_{i 2}, \psi_{i 3}-\sigma_{i}\left(c_{3}\right) \psi_{i 0}, \ldots, \psi_{i n}-\sigma_{i}\left(c_{n}\right) \psi_{i 0}\right), 1 \leq i \leq s$, and let $\widetilde{\Psi_{i}}$ be the strict transform of $\widetilde{\Psi_{i} \odot T} \odot \tau$ via $\left[\Gamma_{c}, \rho\right]$. Then:

- if $\widetilde{\Psi_{i}} \equiv 0$, the series $\psi_{i 0}, \psi_{i 1}, \ldots, \psi_{i n}$ share a factor which is not a unit;

- if $\widetilde{\Psi_{i}} \not \equiv 0$, each nonzero component of $\widetilde{\Psi_{i}}$ is a unit times a power of $x_{2}$ and some component is in fact a unit $\left.\widetilde{\left(\Psi_{i}\right.}(0) \neq 0\right)$.

Proof. First, by 4.1] there exist an ordering $\alpha$ of $k$ and a sequence $T=\left[\varphi_{1}, \ldots, \varphi_{r}\right]$ with coefficients in the real closure $R$ of $(k, \alpha)$ and a series $h \in R\left[\left[x_{2}, \ldots, x_{n}\right]\right]$ of order $\geq 2$ such that $\widetilde{f \odot T}=\left(x_{1}-h\right) U$, where $U \in R\left[\left[x_{1}, \ldots, x_{n}\right]\right]$ is a unit. Choose a finite normal extension $L \supset k_{0}$ contained in $R[\sqrt{-1}]$ such that the sequence $T$ is defined over $L \cap R$. We will see that $\alpha, R, L, T$ and $h$ satisfy the assertions in Proposition 4.4.

In view of 3.3 , there exist finitely many polynomials $q_{1}, \ldots, q_{\ell} \in R\left[x_{1}, \ldots, x_{n}\right]$ such that for every $g \in R\left[\left[x_{1}, \ldots, x_{n}\right]\right]$

$$
g \odot T=q_{1}^{\nu_{1}} \cdots q_{\ell}^{\nu_{\ell}} \widetilde{g \odot T} \text { for } \nu_{1}, \ldots, \nu_{\ell} \geq 0
$$

and $\operatorname{gcd}\left(\widetilde{g \odot T}, \prod_{l=1}^{\ell} q_{l}\right)=1$. Therefore, $q_{l}$ is relatively prime to $\widetilde{f \odot T}=\left(x_{1}-h\right) U$ for all $l$, and so $q_{l}^{\prime}:=q_{l} \odot \tau \neq 0$ for all $l$. Furthermore, since $f$ and $\Delta$ are relatively prime, so are $\widetilde{f \odot T}$ and $\widetilde{\Delta \odot T}$. In particular, $x_{1}-h$ does not divide $\widetilde{\Delta \odot T}$, and so $\widehat{\Delta}:=\Delta \odot T \odot \tau \neq 0$.

Let $E, \psi_{i j}, \sigma_{i}$ be given as in Proposition 4.4. Consider for $i=1, \ldots, s$ and $j=3, \ldots, n$ the sets

$$
Z_{i j}:=\left\{\zeta \in E: \operatorname{gcd}\left(\psi_{i j}+\zeta \psi_{i 0}, \psi_{i 2}\right) \neq \operatorname{gcd}\left(\psi_{i j}, \psi_{i 0}, \psi_{i 2}\right)\right\} .
$$

By 4.3, the sets $Z_{i j}$ are finite; hence, the sets $S_{j}:=\bigcup_{i=1}^{s} \sigma_{i}^{-1}\left(Z_{i j}\right)$ are also finite. 
Let $\Gamma_{c}\left(x_{2}, \ldots, x_{n}\right)=\left(x_{2}, x_{3}+c_{3} x_{2}, \ldots, x_{n}+c_{n} x_{2}\right)$ for each $c=\left(c_{3}, \ldots, c_{n}\right)$, and let $\widehat{\psi_{i j}}:=\psi_{i j} \odot T \odot \tau$ for all $i=1, \ldots, s$ and $j=1, \ldots, n$. Now, we proceed in several steps:

Step 1. There exists $c=\left(c_{3}, \ldots, c_{n}\right) \in E^{n-2}$ such that:

(a) $c_{j} \notin S_{j}$ for $j=3, \ldots, n$,

(b) the series $q_{1}^{\prime} \odot \Gamma_{c}, \ldots, q_{\ell}^{\prime} \odot \Gamma_{c}, \widehat{\Delta} \odot \Gamma_{c}$ are all totally regular with respect to $x_{2}$,

(c) the series $\widehat{\psi_{i 1}} \odot \Gamma_{c}, \widehat{\psi_{i 2}} \odot \Gamma_{c}$ are totally regular with respect to $x_{2}$ or are identically zero, and

$\left(\mathrm{d}_{i j}\right)$ the series $\widehat{\psi_{i j}} \odot \Gamma_{c}-\sigma_{i}\left(c_{j}\right) \widehat{\psi_{i 0}} \odot \Gamma_{c}$ is totally regular with respect to $x_{2}$ or is identically zero $(i=1, \ldots, s$ and $j=3, \ldots, n)$.

Let $c \in E^{n-2}$. For any series $F \in L\left[\left[x_{2}, \ldots, x_{n}\right]\right]$, the series $F \odot \Gamma_{c}$ is totally regular with respect to $x_{2}$ if and only if $\operatorname{In}(F)\left(1, c_{3}, \ldots, c_{n}\right) \neq 0$. Therefore, for each of the condictions (a), (b), (c) and $\left(\mathrm{d}_{i j}\right)$ above, the set of $c \in E^{n-2}$ satisfying this condition is open in the $k_{0}$-Zariski topology of $E^{n-2}$. Thus, it suffices to show that each of these conditions is satisfied by at least one $c \in E^{n-2}$. The only case which is not immediate is $\left(\mathrm{d}_{i j}\right)$ when $\omega\left(\widehat{\psi_{i j}}\right)=\omega\left(\widehat{\psi_{i 0}}\right)<\infty$. Write $F_{i j}=\operatorname{In}\left(\widehat{\psi_{i j}}\right)$, $F_{i 0}=\operatorname{In}\left(\widehat{\psi_{i 0}}\right)$ and assume that

$$
F_{i j}(1, c)-\sigma_{i}\left(c_{j}\right) F_{i 0}(1, c)=0
$$

for all $c \in E^{n-2}$. Since $\left.\sigma_{i}\right|_{k_{0}}=\mathrm{id}$, by 4.2 we have

$$
F_{i j}\left(1, x_{3}, \ldots, x_{n}\right)=x_{j} F_{i 0}\left(1, x_{3}, \ldots, x_{n}\right) .
$$

So $(*)$ says that $\left(c_{j}-\sigma_{i}\left(c_{j}\right)\right) F_{i 0}(1, c)=0$ for every $c \in E^{n-2}$. But neither one of the two factors vanishes identically on $E^{n-2}$, so this is a contradiction.

This completes the proof of Step 1. In the rest of the proof we will show that if $c \in E^{n-2}$ satisfies the conditions of Step 1, then the linear change $\Gamma_{c}$ has properties A) and B) of Proposition 4.4 We will start with

Step 2. If $c \in E^{n-2}$ is as in Step 1, then statements A.i), A.ii) and B) hold for $\Gamma_{c}$.

We begin by proving that A.i) and A.ii) hold. First, notice that since the series $q_{1}^{\prime} \odot \Gamma_{c}, \ldots, q_{\ell}^{\prime} \odot \Gamma_{c}$ are totally regular with respect to $x_{2}$, we have that $q_{l}^{\prime} \odot \Gamma_{c} \odot \rho=$ $x_{2}^{\omega\left(q_{l}^{\prime}\right)} V_{l}$, where $V_{l} \in R\left[\left[x_{2}, \ldots, x_{n}\right]\right]$ is a unit, for all $l$. This follows from the Weierstraß Preparation Theorem and the fact that if $P$ is a Weierstraß polynomial totally regular with respect to $x_{2}$ (that is, of degree with respect to $x_{2}$ equal to its order), then $\widetilde{P \odot \rho}$ is a unit.

Let $g \in k[[x]]$ be a power series such that $g \odot T \odot \tau \neq 0$. As we have seen above, there exist positive integers $\nu_{1}, \ldots, \nu_{\ell} \geq 0$ such that $g \odot T=q_{1}^{\nu_{1}} \cdots q_{r}^{\nu_{\ell}}(\widetilde{g \odot T})$. Hence,

$$
g \odot T \odot \tau \odot \Gamma_{c} \odot \rho=\prod_{l=1}^{\ell}\left(q_{l} \odot \tau \odot \Gamma_{c} \odot \rho\right)^{\nu_{l}} \cdot\left(\widetilde{g \odot T} \odot \tau \odot \Gamma_{c} \odot \rho\right)=x_{2}^{d_{g}} U_{g} \widetilde{g},
$$

where $d_{g}=\sum_{l=1}^{\ell} \omega\left(q_{l}^{\prime}\right) \nu_{l}+\omega(\widetilde{g \odot T} \odot \tau)$ and $U_{g}=\prod_{l=1}^{\ell} V_{l}^{\nu_{l}}$. (Recall that $\tilde{g}$ is the strict transform of $\widehat{g \odot T} \odot \tau$ via $\left.\left[\Gamma_{c}, \rho\right]\right)$.

On the other hand, we recall that $T_{c}^{\prime}$ denotes the sequence of transforms obtained by adding $\varphi_{r+1}=\Gamma_{c}^{\prime}, \varphi_{r+2}=\rho^{\prime}$ to $T$. Note that $\tau \odot \Gamma_{c} \odot \rho=\Gamma_{c}^{\prime} \odot \rho^{\prime} \odot \tau^{\prime}$; hence, $T \odot \tau \odot \Gamma_{c} \odot \rho=T \odot \Gamma_{c}^{\prime} \odot \rho^{\prime} \odot \tau^{\prime}=T_{c}^{\prime} \odot \tau^{\prime}$. Note also that, by the definition of the 
strict transform, the series $\widetilde{g}$ and $x_{2}$ are relatively prime. There exist integers $\mu, \nu_{1}, \ldots, \nu_{\ell} \geq 0$ such that

$$
\begin{aligned}
x_{2}^{d_{g}} U_{g} \widetilde{g} & =g \odot T \odot \tau \odot \Gamma_{c} \odot \rho=g \odot T \odot \Gamma_{c}^{\prime} \odot \rho^{\prime} \odot \tau^{\prime} \\
& =\prod_{l=1}^{\ell}\left(q_{l} \odot \Gamma_{c}^{\prime} \odot \rho^{\prime} \odot \tau^{\prime}\right)^{\nu_{l}} \cdot\left(\widetilde{g \odot T} \odot \Gamma_{c}^{\prime} \odot \rho^{\prime} \odot \tau^{\prime}\right) \\
& =\prod_{l=1}^{\ell}\left(q_{l} \odot \tau \odot \Gamma_{c} \odot \rho\right)^{\nu_{l}} \cdot\left(\widetilde{g \odot T} \odot \Gamma_{c}^{\prime} \odot \rho^{\prime} \odot \tau^{\prime}\right) \\
& \left.=x_{2}^{\mu} \prod_{l=1}^{\ell} V_{l}^{\nu_{l}} \cdot \widetilde{\left(g \odot T_{c}^{\prime} \odot\right.} \tau^{\prime}\right)=x_{2}^{\mu} U_{g}\left(\widetilde{g \odot T_{c}^{\prime} \odot} \tau^{\prime}\right) .
\end{aligned}
$$

Then, since $\widetilde{g}$ and $x_{2}$ are relatively prime, we conclude that there exists an integer $\delta_{g} \geq 0$ such that $\widetilde{g \odot T_{c}^{\prime}} \odot \tau^{\prime}=x_{2}^{\delta_{g}} \widetilde{g}$.

Next we prove that $\mathrm{B}$ ) holds. Since for all $i$ the series

$$
\widehat{\psi_{i 1}} \odot \Gamma_{c}, \widehat{\psi_{i 2}} \odot \Gamma_{c}, \widehat{\psi_{i 3}} \odot \Gamma_{c}-\sigma_{i}\left(c_{3}\right) \widehat{\psi_{i 0}} \odot \Gamma_{c}, \ldots, \widehat{\psi_{i n}} \odot \Gamma_{c}-\sigma_{i}\left(c_{n}\right) \widehat{\psi_{i 0}} \odot \Gamma_{c}
$$

are totally regular with respect to $x_{2}$ or are identically 0 , the strict transforms with respect to $\rho$ of the ones which are not zero are again units. Let us see first that for each $i=1, \ldots, s$ we have either $\widetilde{\Psi_{i} \odot T} \odot \tau \equiv 0$ or $\widetilde{\Psi_{i}}(0) \neq 0$. Suppose that $\widetilde{\Psi_{i}}(0)=0$. As one can deduce from our previous assertions, the non-zero coordinates of $\Psi_{i} \odot T \odot \tau \odot \Gamma_{c} \odot \rho$ are the product of a unit of $R\left[\left[x_{2}, \ldots, x_{n}\right]\right]$ times a power of $x_{2}$. This means that if $\widetilde{\Psi_{i}}(0)=0$, then $x_{2}$ divides all the non-zero components of $\widetilde{\Psi_{i}}$. But this is impossible by the definition of the strict transform, and so $\widetilde{\Psi_{i}} \equiv 0$; hence, $\widetilde{\Psi_{i} \odot T} \odot \tau \equiv 0$.

This last fact means that $x_{1}-h$ divides all the components of $\widetilde{\Psi_{i} \odot T}$. Now, since $q_{l}^{\prime}=q_{l} \odot \tau \neq 0$ for all $l$, we conclude that $x_{1}-h$ divides the strict transforms via $T$ of all the components of $\Psi_{i}$. By [3.2(ii), we deduce that the power series

$$
\psi_{i 1}, \psi_{i 2}, \psi_{i 3}-\sigma_{i}\left(c_{3}\right) \psi_{i 0}, \ldots, \psi_{i n}-\sigma_{i}\left(c_{n}\right) \psi_{i 0},
$$

which are the components of $\Psi_{i}$, share an irreducible factor.

Moreover, since $c_{j} \notin S_{j}$ for $j=3, \ldots, n$, we have in particular $\sigma_{i}\left(c_{j}\right) \notin Z_{i j}$ for all $j=3, \ldots, n$. Thus,

$$
\operatorname{gcd}\left(\psi_{i j}+\sigma_{i}\left(c_{j}\right) \psi_{i 0}, \psi_{i 2}\right)=\operatorname{gcd}\left(\psi_{i j}, \psi_{i 0}, \psi_{i 2}\right)
$$

for all $j=3, \ldots, n$. Hence,

$$
\begin{aligned}
\operatorname{gcd} & \left(\psi_{i 1}, \psi_{i 2}, \psi_{i 3}-\sigma_{i}\left(c_{3}\right) \psi_{i 0}, \ldots, \psi_{i n}-\sigma_{i}\left(c_{n}\right) \psi_{i 0}\right) \\
& =\operatorname{gcd}\left(\psi_{i 1}, \operatorname{gcd}\left(\psi_{i 2}, \psi_{i 3}-\sigma_{i}\left(c_{3}\right) \psi_{i 0}, \ldots, \psi_{i n}-\sigma_{i}\left(c_{n}\right) \psi_{i 0}\right)\right) \\
& =\operatorname{gcd}\left(\psi_{i 1}, \operatorname{gcd}\left(\psi_{i 0}, \psi_{i 2}, \psi_{i 3}, \ldots, \psi_{i n}\right)\right) \\
& =\operatorname{gcd}\left(\psi_{i 0}, \psi_{i 1}, \ldots, \psi_{i n}\right)
\end{aligned}
$$

and we conclude that the series $\psi_{i 0}, \psi_{i 1}, \ldots, \psi_{i n}$ share an irreducible factor.

Step 3. If $c \in E^{n-2}$ is as in Step 1, then statement A.iii) holds for $\Gamma_{c}$.

We recall that $\widehat{\Delta}$ denotes $\Delta \odot T \odot \tau$ and that this series is totally regular with respect to $x_{2}$. Hence, again, $\widehat{\Delta} \odot \Gamma_{c} \odot \rho=x_{2}^{\omega(\widehat{\Delta})} W$, where $W \in R\left[\left[x_{2}, \ldots, x_{n}\right]\right]$ is a unit. Again, let $g \in k[[x]]$ be a power series such that $g \odot T \odot \tau \neq 0$. By A.i), there 
exist an integer $d_{g} \geq 0$ and a unit $U_{g} \in R\left[\left[x_{2}, \ldots, x_{n}\right]\right]$ such that $g \odot T \odot \tau \odot \Gamma_{c} \odot \rho=$ $x_{2}^{d_{g}} U_{g} \widetilde{g}$. Let $\mu:=d_{g}+2 \omega(\widehat{\Delta})$ and $\omega_{1}:=\omega(\widetilde{g})+\mu+1>0$.

Assume we have an equation

$$
\Delta^{2} g+\zeta=h_{1}^{2}+\cdots+h_{p}^{2}+f Q, \quad \text { where } g, h_{1}, \ldots, h_{p}, Q, \zeta \in k[[x]]
$$

with $\omega(\zeta) \geq \omega_{1}$. Let $\lambda_{\zeta}:=\omega\left(\zeta \odot T \odot \tau \odot \Gamma_{c}\right)$. If $\zeta \odot T \odot \tau=0$, take $\xi=0$ (in particular, this happens if $\zeta=0)$. Otherwise, there exists a series $\xi_{1} \in R\left[\left[x_{2}, \ldots, x_{n}\right]\right]$ such that $\zeta \odot T \odot \tau \odot \Gamma_{c} \odot \rho=x_{2}^{\lambda_{\zeta}} \xi_{1}$ and $x_{2}$ does not divide $\xi_{1}$. Noting that $\lambda_{\zeta} \geq \omega(\zeta) \geq$ $\omega_{1}$, we put $\xi:=x_{2}^{\lambda_{\zeta}-\mu}\left(U_{g} W^{2}\right)^{-1} \xi_{1}$. Then $\omega(\xi) \geq \lambda_{\zeta}-\mu \geq \omega_{1}-\mu=\omega(\widetilde{g})+1$, and

$$
\zeta \odot T \odot \tau \odot \Gamma_{c} \odot \rho=x_{2}^{\mu} U_{g} W^{2} \xi
$$

by definition. If we plug $T$ into equation $(\star)$, we obtain

$$
\begin{aligned}
(\Delta \odot T)^{2}(g \odot T)+\zeta \odot T & =\left(h_{1}^{\prime}\right)^{2}+\cdots+\left(h_{p}^{\prime}\right)^{2}+(\widetilde{f \odot T}) Q^{\prime} \\
& =\left(h_{1}^{\prime}\right)^{2}+\cdots+\left(h_{p}^{\prime}\right)^{2}+\left(x_{1}-h\left(x_{2}, \ldots, x_{n}\right)\right) U Q^{\prime}
\end{aligned}
$$

with $h_{1}^{\prime}, \ldots, h_{p}^{\prime}, Q^{\prime} \in R[[x]]$. If we make the substitution $\tau: x_{1}=h$ and plug the sequence $\left[\Gamma_{c}, \rho\right]$ into the previous equation, we get

$$
\begin{array}{r}
\left(\widehat{\Delta} \odot \Gamma_{c} \odot \rho\right)^{2}\left(g \odot T \odot \tau \odot \Gamma_{c} \odot \rho\right)+\zeta \odot T \odot \tau \odot \Gamma_{c} \odot \rho \\
\quad=\left(h_{1}^{\prime} \odot \tau \odot \Gamma_{c} \odot \rho\right)^{2}+\cdots+\left(h_{p}^{\prime} \odot \tau \odot \Gamma_{c} \odot \rho\right)^{2} .
\end{array}
$$

Note that the left hand side is $x_{2}^{\mu} W^{2} U_{g}(\widetilde{g}+\xi)$. Thus, there exist series $a_{1}, \ldots, a_{p} \in$ $R\left[\left[x_{2}, \ldots, x_{n}\right]\right]$ and $\varepsilon= \pm 1$ such that

$$
x_{2}^{\mu} \varepsilon(\widetilde{g}+\xi)=a_{1}^{2}+\cdots+a_{p}^{2} \quad\left(\text { resp. } x_{2}^{\mu} \varepsilon \widetilde{g}=a_{1}^{2}+\cdots+a_{p}^{2}, \quad \text { if } \zeta=0\right) .
$$

It follows that $x_{2}^{\mu}$ divides $a_{i}^{2}$ for each $i$, and we are done.

\section{Proof of the Main Theorem}

The purpose of this section is to prove our Main Theorem 1.1 First, we need some preliminary results:

Lemma 5.1. Let $K$ be a field and $P \in K[x]=K\left[x_{1} \ldots, x_{n}\right]$ a homogeneous psd polynomial. Let $T$ be a sequence of transforms with coefficients in $K$ and let $P_{1}=\left(P \odot T^{-1}\right)^{\vee} \in K[x]$ be a reduced polynomial transform of $P$. Then $P_{1}$ is psd in $K[x]$.

Proof. Let $\Phi$ be the $K$-automorphism of $K(x)$ induced by $T$, see (3.4). Since $d=\operatorname{deg}(P)$ is even, it follows from $P_{1}=g^{d} \Phi^{-1}(P)$ that $P_{1}$ is psd in $K(x)$, hence in $K[x]$.

Lemma 5.2. Let $K \subset E$ be a finite separable field extension and let $P_{1} \in E[x]=$ $E\left[x_{1}, \ldots, x_{n}\right]$ be a psd polynomial. Let $L$ be the Galois hull of $K \subset E, G:=$ $\operatorname{Gal}(L \mid K)$ and $H:=\left\{\sigma \in G:\left.\sigma\right|_{E}=\mathrm{id}\right\}$. Write $G=\bigcup_{i=0}^{s} \sigma_{i} H$ as disjoint union of cosets of $H$ (where $\sigma_{0}=\mathrm{id}$ ). Then $P=P_{1}^{\sigma_{0}} \cdots P_{1}^{\sigma_{s}} \in K[x]$ is psd in $K[x]$.

Proof. First, note that $P$ is the $E \mid K$-norm of $P_{1}$. It is generally true for any finite étale algebra $A \rightarrow B$ that $N_{B \mid A}$ maps psd elements of $B$ to psd elements of $A$. (For the proof one immediately reduces to the case where $A$ is a real closed field, and then the statement is obvious). 
The following is a modest generalization of [CDLR, Thm. 4.10], which is the key construction by which [CDLR] proved the infinity of the Pythagoras number for many rings:

Lemma 5.3. Let $R$ be a real closed field. For every $r, p \geq 2$ we define the polynomials $\Delta_{1}=x_{2}, \Delta_{r}=x_{2} \prod_{s=2}^{r}\left(x_{2}-s x_{1}\right) \in \mathbb{Z}\left[x_{1}, x_{2}\right], f_{1}=y_{1}^{2}$ and

$$
f_{p}\left(y_{1}, \ldots, y_{p} ; x_{1}, x_{2}\right)=y_{p}^{2}+\sum_{j=2}^{p} y_{p-j+1}^{2} \prod_{k=2}^{j} \Delta_{2^{p-k}}^{2} .
$$

Then for every $a_{1}, \ldots, a_{p} \in R \backslash\{0\}$ we have that $f_{p}\left(a_{1}, \ldots, a_{p} ; x_{1}, x_{2}\right)$ is a sum of $p$ squares in $R\left[x_{1}, x_{2}\right]$ but it is not a sum of $p-1$ squares.

Proof. The proof of this result is done using induction. First, note that the $f_{p}$ can be defined recursively in the following way:

$$
\begin{array}{cccc}
f_{1}=y_{1}^{2}, & & \\
f_{2}=y_{1}^{2} \Delta_{1}^{2}+y_{2}^{2} & = & f_{1} \Delta_{1}^{2}+y_{2}^{2}, \\
f_{3}=y_{1}^{2} \Delta_{1}^{2} \Delta_{2}^{2}+y_{2}^{2} \Delta_{2}^{2}+y_{3}^{2} & = & f_{2} \Delta_{2}^{2}+y_{3}^{2}, \\
f_{4}=y_{1}^{2} \Delta_{1}^{2} \Delta_{2}^{2} \Delta_{4}^{2}+y_{2}^{2} \Delta_{2}^{2} \Delta_{4}^{2}+y_{3}^{2} \Delta_{4}^{2}+y_{4}^{2} & = & f_{3} \Delta_{4}^{2}+y_{4}^{2}, \\
\vdots & \vdots & & \vdots \\
f_{p}=\sum_{j=2}^{p} y_{p-j+1}^{2} \prod_{k=2}^{j} \Delta_{2^{p-k}}^{2}+y_{p}^{2} & = & f_{p-1} \Delta_{2^{p-2}}^{2}+y_{p}^{2}, \\
\vdots & \vdots & & \vdots
\end{array}
$$

Notice that for every $p, q \geq 1$ and $a_{1}, \ldots, a_{p} \in R \backslash\{0\}$ the polynomial

$$
f_{p}\left(a_{1}, \ldots, a_{p} ; x_{1}, x_{2}\right)
$$

is a sum of $p$ squares and that $f_{p}\left(a_{1}, \ldots, a_{p} ; x_{1}, x_{2}\right)$ is a sum of $q$ squares if and only if the polynomial

$$
\frac{1}{a_{p}^{2}} f_{p}\left(a_{1}, \ldots, a_{p} ; x_{1}, x_{2}\right)=f_{p}\left(\frac{a_{1}}{a_{p}}, \ldots, \frac{a_{p-1}}{a_{p}}, 1 ; x_{1}, x_{2}\right)
$$

is a sum of $q$ squares. Therefore, it is enough to show that for $a_{1}, \ldots, a_{p-1} \in R \backslash\{0\}$ the polynomial $f_{p}\left(a_{1}, \ldots, a_{p-1}, 1 ; x_{1}, x_{2}\right)$ is not a sum of $p-1$ squares. This is clear for $p=1$, so we can suppose $p>1$. Using induction on $p$ (and the previous remark), we may assume that $f_{p-1}\left(a_{1}, \ldots, a_{p-1} ; x_{1}, x_{2}\right)$ is not a sum of $p-2$ squares. Now, proceeding analogously to the proofs of [PD, 8.1.2] or [CDLR, 4.10-11,16], we conclude that if there exist $a_{1}, \ldots, a_{p-1} \in R \backslash\{0\}$ such that the polynomial $f_{p}\left(a_{1}, \ldots, a_{p-1}, 1 ; x_{1}, x_{2}\right)$ is a sum of $p-1$ squares, then $f_{p-1}\left(a_{1}, \ldots, a_{p-1} ; x_{1}, x_{2}\right)$ is a sum of $p-2$ squares, against the induction hypothesis.

Now we are finally ready to prove 1.2

Proof of Theorem 1.2. First, by (2.1) we can suppose that $\mathfrak{Q}$ is properly immersed. Let $B=k\left[\left[x_{1}, \ldots, x_{N}\right]\right] / \mathfrak{Q}$, which is a real reduced domain of dimension $d \geq$ 3. By local parametrization (2.1) there is an irreducible Weierstraß polynomial $f \in k\left[\left[x_{1}, \ldots, x_{d}\right]\right]\left[x_{d+1}\right]$ whose degree $p$ is equal to its order, with discriminant $\Delta \in k\left[\left[x_{1}, \ldots, x_{d}\right]\right]$, such that $(f)=\mathfrak{Q} \cap K\left[\left[x_{1}, \ldots, x_{d}\right]\right]\left[x_{d+1}\right]$. The canonical homomorphism $A=k\left[\left[x_{1}, \ldots, x_{d}\right]\right] \rightarrow B$ is injective and finite. Moreover, if $\theta_{d+1}$ is the class of $x_{d+1} \bmod \mathfrak{Q}$, then

$$
\Delta \cdot B \subset A+A \theta_{d+1}+\cdots+A \theta_{d+1}^{p-1} \cong B^{\prime},
$$


where $B^{\prime}=k\left[\left[x_{1}, \ldots, x_{d}, x_{d+1}\right]\right] /(f)$. Note also that $B^{\prime}$ is a real reduced domain, since $B$ is one and $B^{\prime} \hookrightarrow B$. Furthermore, since $\mathfrak{Q}$ is properly immersed, by 2.2 there exists an integer $\Lambda>0$ such that for all $g \in k\left[\left[x_{1}, \ldots, x_{N}\right]\right]$ there exists a polynomial $g^{\prime} \in k\left[\left[x_{1}, \ldots, x_{d}\right]\right]\left[x_{d+1}\right]$ of degree $\leq p-1$ and order $\geq \omega(g)-\Lambda$ such that $\Delta g \equiv g^{\prime} \bmod \mathfrak{Q}$.

Let $n=d+1$ and $\alpha, R, L, T, h$ be as in 4.4 for the power series $f, \Delta$. In what follows we denote

$$
\tau\left(x_{2}, \ldots, x_{n}\right):=\left(h, x_{2}, \ldots, x_{n}\right)
$$

and

$$
\rho\left(x_{2}, \ldots, x_{n}\right):=\left(x_{2}, x_{2} x_{3}, \ldots, x_{2} x_{n}\right) .
$$

We write $T^{-1}=\left(\frac{f_{1}}{g}, \ldots, \frac{f_{n}}{g}\right)$, where $f_{1}, \ldots, f_{n}, g \in(L \cap R)[x]$ are relatively prime, and consider the subgroup of $G=\operatorname{Gal}\left(L \mid k_{0}\right)$ given by $H=\{\sigma \in G$ : $\left.\left(f_{1}^{\sigma}, \ldots, f_{n}^{\sigma}, g^{\sigma}\right)=\left(f_{1}, \ldots, f_{n}, g\right)\right\}$. Let $E \subset L \cap R$ be the fixed field associated to $H$. Write $G=\bigcup_{i=0}^{s} \sigma_{i} H$ as a disjoint union of cosets of $H$, where $\sigma_{0}=$ id. For each $c=\left(c_{3}, \ldots, c_{n}\right) \in E^{n-2}$ consider the sequence of transforms $T_{c}^{\prime}=\left[\varphi_{1}, \ldots, \varphi_{r+2}\right]$ obtained by adding to $T$ the transforms

$$
\varphi_{r+1}=\Gamma_{c}^{\prime}:=\left(x_{1}, \Gamma_{c}\left(x_{2}, \ldots, x_{n}\right)\right)=\left(x_{1}, x_{2}, x_{3}+c_{3} x_{2}, \ldots, x_{n}+c_{n} x_{2}\right)
$$

and

$$
\varphi_{r+2}=\rho^{\prime}:=\left(x_{1}, \rho\left(x_{2}, \ldots, x_{n}\right)\right)=\left(x_{1}, x_{2}, x_{2} x_{3}, \ldots, x_{2} x_{n}\right) .
$$

Then, we have

$$
\begin{aligned}
\left(T_{c}^{\prime}\right)^{-1}=\left(\frac{f_{1}}{g}, \frac{f_{2}}{g}, \frac{f_{3}-c_{3} f_{2}}{f_{2}}\right. & \left., \ldots, \frac{f_{n}-c_{n} f_{2}}{f_{2}}\right) \\
& =\left(\frac{f_{1} f_{2}}{g f_{2}}, \frac{f_{2}^{2}}{g f_{2}}, \frac{g\left(f_{3}-c_{3} f_{2}\right)}{g f_{2}}, \ldots, \frac{g\left(f_{n}-c_{n} f_{2}\right)}{g f_{2}}\right) .
\end{aligned}
$$

Hence, there exist polynomials $F_{0}, F_{1}, \ldots, F_{n} \in E[x]$, not depending on $c$, such that

$$
\left(T_{c}^{\prime}\right)^{-1}=\left(\frac{F_{1}}{F_{0}}, \frac{F_{2}}{F_{0}}, \frac{F_{3}-c_{3} F_{0}}{F_{0}}, \ldots, \frac{F_{n}-c_{n} F_{0}}{F_{0}}\right)
$$

and $\operatorname{gcd}\left(F_{0}, F_{1}, \ldots, F_{n}\right)=1$.

Let $\psi_{i j}:=F_{j}^{\sigma_{i}}$ for $i=1, \ldots, s$ and $j=0, \ldots, n$, and let $c \in E^{n-2}$ and $\Gamma_{c}$ be as in 4.4 for the field $E$, the series $\psi_{i j}$ and the automorphisms $\sigma_{i}$. Since $\operatorname{gcd}\left(F_{0}, F_{1}, \ldots, F_{n}\right)=1$, we also have $\operatorname{gcd}\left(F_{0}^{\sigma_{i}}, F_{1}^{\sigma_{i}}, \ldots, F_{n}^{\sigma_{i}}\right)=1$ for $i=1, \ldots, s$. Let

$$
\Psi_{i}:=\left(F_{1}^{\sigma_{i}}, F_{2}^{\sigma_{i}}, F_{3}^{\sigma_{i}}-\sigma_{i}\left(c_{3}\right) F_{0}^{\sigma_{i}}, \ldots, F_{n}^{\sigma_{i}}-\sigma_{i}\left(c_{n}\right) F_{0}^{\sigma_{i}}\right)
$$

for $i=0, \ldots, s$. Note that $\Psi_{i}=\Psi_{0}^{\sigma_{i}}$ for all $i$. On the other hand, if $\widetilde{\Psi_{i}}$ is the strict transform of $\widetilde{\Psi_{i} \odot T} \odot \tau$ via $\left[\Gamma_{c}, \rho\right]$ for $i=1, \ldots, s$, then, by $4.4 \mathrm{~B}$ ), we have $\widetilde{\Psi_{i}} \not \equiv 0$. Moreover, each nonzero component of $\widetilde{\Psi_{i}}$ is the product of a unit and a power of $x_{2}$, and $\widetilde{\Psi_{i}}(0) \neq 0$. Hence, using 4.4 A.i $)$, it follows that for $i=1, \ldots, s$ there exist an integer $d_{i} \geq 0$ and a unit $U_{i} \in R\left[\left[x_{2}, \ldots, x_{n}\right]\right]$ such that

$$
\Psi_{i} \odot T \odot \tau \odot \Gamma_{c} \odot \rho=x_{2}^{d_{i}} U_{i} \widetilde{\Psi_{i}} .
$$

Next, let us prove the existence of polynomials $M, N_{p} \in k_{0}[x], p \geq 1$, such that:

(i) $M$ is psd in $k_{0}[x]$ and there exists an integer $\omega_{0} \geq 0$ such that if $\omega(\zeta) \geq \omega_{0}$ then $M+\zeta$ is not a sum of squares in $k\left[\left[x_{1}, \ldots, x_{N}\right]\right] / \mathfrak{Q}$; and 
(ii) $N_{p}$ is a sum of $p$ squares in $k_{0}[x]$ but it is not a sum of $p-1$ squares in $k\left[\left[x_{1}, \ldots, x_{N}\right]\right] / \mathfrak{Q}$.

For that, we proceed in several steps. In what follows, given a series $g \in R[[x]]$, we will denote by $\widetilde{g}$ the strict transform of $\widetilde{g \odot T} \odot \tau$ via $\left[\Gamma_{c}, \rho\right]$.

Step 1. Key construction to prove the existence of $M:$ Let $P_{0} \in k_{0}\left[x^{\prime}\right]=$ $k_{0}\left[x_{2}, \ldots, x_{n}\right]$ be a homogeneous polynomial of degree $m$. There exist a polynomial $P \in k_{0}[x]$, a linear change $\pi$ in $k_{0}^{n-1}$ and a value $u_{0} \in R \backslash\{0\}$ such that $\operatorname{In}(\widetilde{P})=u_{0} \cdot P_{0} \odot \pi$. Moreover, if $P_{0}$ is psd in $k_{0}\left[x^{\prime}\right]$, then $P$ is also psd in $k_{0}[x]$.

Indeed, let $\pi\left(x^{\prime}\right)$ be a linear change in $k_{0}^{n-1}$ (with coefficients in $k_{0}$ ) and $v \in k_{0}^{n-1}$ such that $x_{2}$ does not divide $P_{0} \odot \pi$ and $\left(P_{0} \odot \pi^{\prime}\right)\left(\widetilde{\Psi_{i}}(0)\right) \neq 0$ for all $i$, where $\pi^{\prime}: k_{0}^{n} \rightarrow$ $k_{0}^{n-1}$ is defined by $\pi^{\prime}(x):=\pi\left(x^{\prime}\right)+x_{1} v$. Let $P_{1}:=\left(\left(P_{0} \odot \pi^{\prime}\right) \odot\left(T_{c}^{\prime}\right)^{-1}\right)^{\vee}=P_{0} \odot \pi^{\prime} \odot \Psi_{0}$ and

$$
P:=P_{1}^{\sigma_{0}} \cdots P_{1}^{\sigma_{s}}=P_{1} P_{1}^{\sigma_{1}} \cdots P_{1}^{\sigma_{s}} \in k_{0}[x] .
$$

Note that if $P_{0}$ is psd in $k_{0}\left[x^{\prime}\right]$, hence in $k_{0}[x]$, then, by 5.1, the polynomial $P_{1}$ is psd in $E[x]$ and, by 5.2 the polynomial $P$ is psd in $k_{0}[x]$. Moreover, we have

$$
P_{1}^{\sigma_{i}}=\left(P_{0} \odot \pi^{\prime} \odot \Psi_{0}\right)^{\sigma_{i}}=P_{0} \odot \pi^{\prime} \odot \Psi_{0}^{\sigma_{i}}=P_{0} \odot \pi^{\prime} \odot \Psi_{i}, \text { for } i=0, \ldots, s
$$

and, by $(3.4), P_{1} \odot T_{c}^{\prime}=\left(F_{0} \odot T_{c}^{\prime}\right)^{m}\left(P_{0} \odot \pi^{\prime}\right)$.

Next, by 3.5 we have $\widehat{F_{0} \odot T_{c}^{\prime}}=u \in R \backslash\{0\}$. We also recall that if $\tau^{\prime}:=$ $\left(h^{\prime}, x_{2}, \ldots, x_{n}\right)$, where $h^{\prime}:=h \odot \Gamma_{c} \odot \rho$, then $\tau \odot \Gamma_{c} \odot \rho=\Gamma_{c}^{\prime} \odot \rho^{\prime} \odot \tau^{\prime}$. Hence,

$$
T \odot \tau \odot \Gamma_{c} \odot \rho=T \odot \Gamma_{c}^{\prime} \odot \rho^{\prime} \odot \tau^{\prime}=T_{c}^{\prime} \odot \tau^{\prime} .
$$

Let $\widetilde{F_{0}}$ be the strict transform of $\widetilde{F_{0} \odot T} \odot \tau$ via $\left[\Gamma_{c}, \rho\right]$. By $\left.4.4 \mathrm{~A} . \mathrm{i}\right)$, there exist a unit $U_{0}^{\prime} \in R\left[\left[x_{2}, \ldots, x_{n}\right]\right]$ and an integer $d_{0} \geq 0$ such that $x_{2}^{d_{0}} U_{0}^{\prime} \widetilde{F_{0}}=F_{0} \odot T \odot \tau \odot \Gamma_{c} \odot \rho=$ $F_{0} \odot T_{c}^{\prime} \odot \tau^{\prime}$. By A.ii), there exists an integer $\delta_{0} \geq 0$ such that $u=\widehat{F_{0} \odot T_{c}^{\prime} \odot \tau^{\prime}}=$ $x_{2}^{\delta_{0}} \widetilde{F_{0}}$. Hence $\widetilde{F_{0}}=u$, and we get

$$
F_{0} \odot T \odot \tau \odot \Gamma_{c} \odot \rho=x_{2}^{d_{0}} U_{0},
$$

where $U_{0} \in R\left[\left[x_{2}, \ldots, x_{n}\right]\right]$ is the unit $u U_{0}^{\prime}$. Again by 4.4 A.i), there exist a unit $U_{P}$ and an integer $d_{P} \geq 0$ such that $x_{2}^{d_{P}} U_{P} \widetilde{P}=P \odot T \odot \tau \odot \Gamma_{c} \odot \rho$. Computing a little, we have

$$
\begin{aligned}
x_{2}^{d_{P}} U_{P} \widetilde{P} & =P \odot T \odot \tau \odot \Gamma_{c} \odot \rho=P \odot T \odot \Gamma_{c}^{\prime} \odot \rho^{\prime} \odot \tau^{\prime}=P \odot T_{c}^{\prime} \odot \tau^{\prime} \\
& =\left(P_{1} \odot T_{c}^{\prime} \odot \tau^{\prime}\right) \prod_{i=1}^{s}\left(P_{1}^{\sigma_{i}} \odot T_{c}^{\prime} \odot \tau^{\prime}\right) \\
& =\left(F_{0} \odot T_{c}^{\prime} \odot \tau^{\prime}\right)^{m}\left(P_{0} \odot \pi^{\prime} \odot \tau^{\prime}\right) \prod_{i=1}^{s}\left(P_{1}^{\sigma_{i}} \odot T \odot \tau \odot \Gamma_{c} \odot \rho\right) \\
& =\left(F_{0} \odot T_{c}^{\prime} \odot \tau^{\prime}\right)^{m}\left(P_{0} \odot \pi^{\prime} \odot \tau^{\prime}\right) \prod_{i=1}^{s}\left(P_{0} \odot \pi^{\prime} \odot \Psi_{i} \odot T \odot \tau \odot \Gamma_{c} \odot \rho\right) \\
& =\left(F_{0} \odot T_{c}^{\prime} \odot \tau^{\prime}\right)^{m}\left(P_{0} \odot \pi^{\prime} \odot \tau^{\prime}\right) \prod_{i=1}^{s}\left(P_{0} \odot \pi^{\prime}\left(x_{2}^{d_{1}} U_{i} \widetilde{\Psi_{i}}\right)\right) \\
& =x_{2}^{m\left(d_{0}+\cdots+d_{s}\right)} U_{0}^{m} U_{1}^{m} \cdots U_{s}^{m}\left(P_{0} \odot \pi^{\prime} \odot \tau^{\prime}\right) \prod_{i=1}^{s}\left(P_{0} \odot \pi^{\prime}\left(\widetilde{\Psi_{i}}\right)\right) .
\end{aligned}
$$


Since $\left(P_{0} \odot \pi^{\prime}\right)\left(\widetilde{\Psi_{i}}(0)\right) \neq 0$ for $i=1, \ldots, s$, we deduce that every factor $P_{0} \odot \pi^{\prime}\left(\widetilde{\Psi_{i}}\right)$ is a unit. Since $\omega\left(h^{\prime}\right) \geq 2$, we have $\operatorname{In}\left(P_{0} \odot \pi^{\prime} \odot \tau^{\prime}\right)=\operatorname{In}\left(P_{0}\left(\pi\left(x^{\prime}\right)+h^{\prime} v\right)\right)=P_{0} \odot \pi$. Since $x_{2}$ divides neither $\widetilde{P}$ nor $P_{0} \odot \pi=\operatorname{In}\left(P_{0} \odot \pi^{\prime} \odot \tau^{\prime}\right)$, we conclude that:

(1) There exists a unit $U_{P_{0}} \in R[[x]]$ such that $\widetilde{P}=U_{P_{0}} \cdot P_{0} \odot \pi^{\prime} \odot \tau^{\prime}$. Hence, there exists a value $u_{0} \in R \backslash\{0\}$ such that $\operatorname{In}(\widetilde{P})=u_{0} \cdot \operatorname{In}\left(P_{0} \odot \pi^{\prime} \odot \tau^{\prime}\right)=u_{0} \cdot\left(P_{0} \odot \pi\right)$.

(2) In addition, $d_{P}=m\left(d_{0}+\cdots+d_{m}\right)=d(m)$ only depends on $m=\operatorname{deg}\left(P_{0}\right)$.

Step 2. Key construction to prove the existence of $N_{p}$ : Let $Q_{01}, \ldots, Q_{0 p} \in$ $k_{0}\left[x^{\prime}\right]=k_{0}\left[x_{2}, \ldots, x_{n}\right]$ be homogeneous polynomials of the same degree $m$. There exist a polynomial $Q \in k_{0}[x]$ which is a sum of $p$ squares in $k_{0}[x]$, a linear change $\pi$ in $k_{0}^{n-1}$ and values $u_{0}, u_{1}, \ldots, u_{p} \in R \backslash\{0\}$ such that $\operatorname{In}(\widetilde{Q})=u_{0}\left(u_{1} \cdot Q_{01} \odot \pi\right)^{2}+$ $\cdots+u_{0}\left(u_{1} \cdot Q_{0 p} \odot \pi\right)^{2}$.

Let a linear change $\pi\left(x^{\prime}\right)$ in $k_{0}^{n-1}$ (with coefficients in $k_{0}$ ) and $v \in k_{0}^{n-1}$ be such that $x_{2}$ does not divide $Q_{0 \ell} \odot \pi$ and $\left(Q_{0 \ell} \odot \pi^{\prime}\right)\left(\widetilde{\Psi_{i}}(0)\right) \neq 0$ for all $i, \ell$, where $\pi^{\prime}: k_{0}^{n} \rightarrow k_{0}^{n-1}$ is defined by $\pi^{\prime}(x):=\pi\left(x^{\prime}\right)+x_{1} v$. Perform the construction in Step 1, with this $\pi^{\prime}$, for each $P_{0}=Q_{0 \ell}$, and denote in each case by $Q_{\ell}$ the final polynomial $P$ obtained. Let $Q:=Q_{1}^{2}+\cdots+Q_{p}^{2} \in k_{0}[x]$, which is a sum of $p$ squares in $k_{0}[x]$.

By 4.4 A.i), there are units $U_{Q_{\ell}}$ and integers $d_{Q_{\ell}} \geq 0$ such that

$$
x_{2}^{d_{Q_{\ell}}} U_{Q_{\ell}} \widetilde{Q_{\ell}}=Q_{\ell} \odot T \odot \tau \odot \Gamma_{c} \odot \rho .
$$

As we have seen in the proof of Step 1, the integer $d_{Q_{\ell}}$ only depends on $\operatorname{deg} Q_{0 \ell}=m$. Hence, $d_{Q_{1}}=\cdots=d_{Q_{p}}=d$. Moreover, we have also seen that there exist values $u_{0 \ell} \in R \backslash\{0\}$ such that $\operatorname{In}\left(\widetilde{Q_{\ell}}\right)=u_{0 \ell} \cdot\left(Q_{0 \ell} \odot \pi\right)$. Again by 4.4 A.i $)$, there are a unit $U_{Q}$ and an integer $d_{Q} \geq 0$ such that $x_{2}^{d_{Q}} U_{Q} \widetilde{Q}=Q \odot T \odot \tau \odot \Gamma_{c} \odot \rho$. Thus, we have

$$
\begin{aligned}
x_{2}^{d_{Q}} U_{Q} \widetilde{Q}= & Q \odot T \odot \tau \odot \Gamma_{c} \odot \rho \\
& =\sum_{\ell=1}^{p}\left(Q_{\ell} \odot T \odot \tau \odot \Gamma_{c} \odot \rho\right)^{2}=\sum_{\ell=1}^{p}\left(x_{2}^{d_{Q_{\ell}}} U_{Q_{\ell}} \widetilde{Q_{\ell}}\right)^{2}=x_{2}^{d} \sum_{\ell=1}^{p}\left(U_{Q_{\ell}} \widetilde{Q_{\ell}}\right)^{2} .
\end{aligned}
$$

Since $x_{2}$ divides neither $\widetilde{Q}$ nor $\sum_{\ell=1}^{p}\left(U_{Q_{\ell}} \widetilde{Q_{\ell}}\right)^{2}$, we deduce that there exists a unit $V \in R\left[\left[x_{2}, \ldots, x_{n}\right]\right]$ such that $\widetilde{Q}=V \sum_{\ell=1}^{p}\left(U_{Q_{\ell}} \widetilde{Q}_{\ell}\right)^{2}$. Hence, comparing initial forms, we conclude that there exist nonzero values $u_{0}, u_{1}, \ldots, u_{p} \in R \backslash\{0\}$ such that $\operatorname{In}(\widetilde{Q})=u_{0}\left(u_{1} \cdot Q_{01} \odot \pi\right)^{2}+\cdots+u_{0}\left(u_{1} \cdot Q_{0 p} \odot \pi\right)^{2}$.

Step 3. Construction of $M$. Let $P_{0}=x_{2}^{6}+x_{3}^{4} x_{4}^{2}+x_{3}^{2} x_{4}^{4}-3 x_{2}^{2} x_{3}^{2} x_{4}^{2}$ be the Motzkin polynomial which is a psd form in $\mathbb{Q}\left[x_{2}, x_{3}, x_{4}\right]$, hence in $k_{0}\left[x^{\prime}\right]=k_{0}\left[x_{2}, \ldots, x_{n}\right]$, but it is not a sum of squares of polynomials over any real closed field [BCR 6.3.6]. By Step 1 there exist a psd polynomial $P \in k_{0}[x]$, a linear change $\pi$ in $k_{0}^{n-1}$ and a value $u_{0} \in R \backslash\{0\}$ such that $\operatorname{In}(\widetilde{P})=u_{0} \cdot P_{0} \odot \pi$. We take $M:=P$.

As for $\omega_{0}$, pick $\omega_{1}$ from 4.4 A.iii) and set $\omega_{0}=\omega_{1}+\Lambda$, where $\Lambda>0$ is the integer introduced at the beginning of this proof which has the property that, if $g \in k\left[\left[x_{1}, \ldots, x_{N}\right]\right]$, then $\Delta g \equiv g^{\prime} \bmod \mathfrak{Q}$ for some $g^{\prime} \in k\left[\left[x_{1}, \ldots, x_{d}\right]\right]\left[x_{d+1}\right]$ of degree $\leq p-1$ and order $\geq \omega(g)-\Lambda$.

If $\omega(\zeta) \geq \omega_{0}$ and $M+\zeta$ was a sum of squares in $B=k\left[\left[x_{1}, \ldots, x_{N}\right]\right] / \mathfrak{Q}$, then, since $\Delta \cdot B \subset B^{\prime}$, the element $\Delta^{2} \cdot(M+\zeta)$ would be a sum of squares in $B^{\prime}$. By the property of $\Lambda$, there exists a polynomial $\zeta^{\prime} \in k\left[\left[x_{1}, \ldots, x_{d}\right]\right]\left[x_{d+1}\right]$ of degree $\leq p-1$ and order $\geq \omega(\zeta)-\Lambda \geq \omega_{0}-\Lambda=\omega_{1}$ such that $\Delta \zeta \equiv \zeta^{\prime} \bmod \mathfrak{Q}$. Thus, $\Delta^{2} M+\Delta \zeta^{\prime}$ would be a sum of squares in $B^{\prime}$, and $\omega\left(\Delta \zeta^{\prime}\right) \geq \omega_{1}$. By 4.4 A.iii), if $\widetilde{M}$ is the strict transform of 
$\widetilde{M \odot T} \odot \tau$ via $\left[\Gamma_{c}, \rho\right]$, there would exist a series $\xi \in R\left[\left[x_{2}, \ldots, x_{n}\right]\right]$ of order $>\omega(\widetilde{M})$ such that either $\widetilde{M}+\xi$ or $-(\widetilde{M}+\xi)$ would be a sum of squares in $R\left[\left[x_{2}, \ldots, x_{n}\right]\right]$. Comparing initial forms, we would have that either $\operatorname{In}(\widetilde{M}+\xi)=\operatorname{In}(\widetilde{M})=u_{0} \cdot P_{0} \odot \pi$ or $-\operatorname{In}(\widetilde{M}+\xi)$ is a sum of squares of (homogeneous) polynomials in $R\left[x_{2}, \ldots, x_{n}\right]$. Hence, we would conclude, after composing with $\pi^{-1}$, that either $P_{0}$ or $-P_{0}$ is a sum of squares of (homogeneous) polynomials in $R\left[x_{2}, \ldots, x_{n}\right]$, a contradiction.

Step 4. Construction of $N_{p}$. For $p=1$ take $N_{p}:=1$, so we can fix $p \geq 2$. Let $f_{p 1}\left(y_{1}, \ldots, y_{p} ; x_{1}, x_{2}\right):=y_{p}^{2}$ and $f_{p \ell}\left(y_{1}, \ldots, y_{p} ; x_{1}, x_{2}\right):=y_{p-\ell+1}^{2} \prod_{i=2}^{\ell} \Delta_{2^{p-i}}^{2}$ where $\Delta_{1}:=x_{2}$ and $\Delta_{2^{p-i}}:=x_{2} \prod_{s=2}^{2^{p-i}}\left(x_{2}-s x_{1}\right) \in \mathbb{Z}\left[x_{1}, x_{2}\right]$ for $\ell=2, \ldots, p$. Then $f_{p}:=f_{1 p}^{2}+\cdots+f_{p p}^{2}$ is the polynomial of 5.3. Let

$$
Q_{0 \ell}:=x_{4}^{\operatorname{deg} f_{p}} f_{p \ell}\left(1, \ldots, 1 ; \frac{x_{2}}{x_{4}}, \frac{x_{3}}{x_{4}}\right)
$$

for $\ell=1, \ldots, p$. The polynomials $Q_{0 \ell} \in \mathbb{Q}\left[x_{2}, x_{3}, x_{4}\right] \subset \mathbb{Q}[x] \subset k_{0}[x]$ are homogeneous of the same degree $\operatorname{deg}\left(f_{p}\right)$. By Step 2 there exist a polynomial $Q \in$ $k_{0}[x]$ which is a sum of $p$ squares in $k_{0}[x]$, a linear change $\pi$ in $k_{0}^{n-1}$ and values $u_{0}, u_{1}, \ldots, u_{p} \in R \backslash\{0\}$ such that $\operatorname{In}(\widetilde{Q})=u_{0}\left(u_{1} \cdot Q_{01} \odot \pi\right)^{2}+\cdots+u_{0}\left(u_{1} \cdot Q_{0 p} \odot \pi\right)^{2}$. We take $N_{p}:=Q$.

We claim that $N_{p}$ is not a sum of $p-1$ squares in $B=k\left[\left[x_{1}, \ldots, x_{N}\right]\right] / \mathfrak{Q}$. Otherwise, since $\Delta \cdot B \subset B^{\prime}$, the element $\Delta^{2} \cdot N_{p}$ would be a sum of $p-1$ squares in $B^{\prime}$. Let $\widetilde{N_{p}}$ denote the strict transform of $\left(\widetilde{N_{p} \odot T}\right) \odot \tau$ via $\left[\Gamma_{c}, \rho\right]$. By 4.4 A.iii), either $\widetilde{N_{p}}$ or $-\widetilde{N}_{p}$ would be a sum of $p-1$ squares in $R\left[\left[x_{2}, \ldots, x_{n}\right]\right]$. Comparing initial forms, we would have that either $\operatorname{In}\left(\widetilde{N_{p}}\right)$ or $-\operatorname{In}\left(\widetilde{N}_{p}\right)$ is a sum of $p-1$ squares of (homogeneous) polynomials in $R\left[x_{2}, \ldots, x_{n}\right]$. Since

$$
\begin{aligned}
\operatorname{In}\left(\widetilde{N_{p}}\right) \odot \pi^{-1} & =u_{0}\left(u_{1} \cdot Q_{01}\right)^{2}+\cdots+u_{0}\left(u_{1} \cdot Q_{0 p}\right)^{2} \\
& =u_{0} x_{4}^{\operatorname{deg} f_{p}} f_{p}\left(u_{1}, \ldots, u_{p} ; \frac{x_{2}}{x_{4}}, \frac{x_{3}}{x_{4}}\right),
\end{aligned}
$$

we would have that either $g_{p}:=f_{p}\left(u_{1}, \ldots, u_{p} ; x_{2}, x_{3}\right)$ or $-g_{p}$ is a sum of $p-1$ squares of polynomials in $R\left[x_{2}, \ldots, x_{n}\right]$. Since $-g_{p}$ cannot be a sum of squares in $R\left[x_{2}, \ldots, x_{n}\right]$, we would conclude that $g_{p}$ is a sum of $p-1$ squares of polynomials in $R\left[x_{2}, \ldots, x_{n}\right]$, which is a contradiction by [5.3. Thus, we have seen that $N_{p}$ is not a sum of $p-1$ squares in $k\left[\left[x_{1}, \ldots, x_{N}\right]\right] / \mathfrak{Q}$, and the proof of $[1.2$ is complete.

Remark 5.4. Let $A$ be an excellent reduced ring of real dimension $d \geq 3$ and let $\operatorname{Quot}(A)$ be its total ring of fractions. Then $p(\operatorname{Quot}(A)) \geq d+1$.

Indeed, since $A$ is reduced and noetherian, we have by $\mathrm{JP}, 1.4 .27]$

$$
\operatorname{Quot}(A)=\bigoplus_{i=1}^{r} \operatorname{Quot}\left(A / \mathfrak{p}_{i}\right)
$$

where the $\mathfrak{p}_{i}$ are the minimal ideals of $A$. Therefore it is enough to consider the case when $A$ is a domain. Now, take an element $R \in \mathcal{P}(A)$ similar to the one in (2.3) but beginning in this case from a polynomial $M \in k_{0}[x]$ such that: $M$ is a sum of $d+1$ squares in $k_{0}(x)$ (hence, psd in $\left.k_{0}[x]\right), M(0)=0$, and there exists an integer $\omega_{0} \geq 0$ such that if $\zeta \in k\left[x_{1}, \ldots, x_{N}\right]$ and $\omega(\zeta) \geq \omega_{0}$, then $M+\zeta$ is not a sum of $d$ squares in $\operatorname{Quot}\left(k\left[\left[x_{1}, \ldots, x_{N}\right]\right] / \mathfrak{Q}\right)$. Since $R \in \mathcal{P}(A)$, we have $R \in \Sigma(\operatorname{Quot}(A))$. If $R$ were a sum of $d$ squares in $\operatorname{Quot}(A)$, we would deduce (proceeding similarly to 
(2.3)) that there exists a series $\zeta \in k\left[\left[x_{1}, \ldots, x_{N}\right]\right]$ with $\omega(\zeta) \geq \omega_{0}$ such that $M+\zeta$ is a sum of $d$ squares in $\operatorname{Quot}\left(k\left[\left[x_{1}, \ldots, x_{N}\right]\right] / \mathfrak{Q}\right)$, a contradiction.

To construct this polynomial $M$, we proceed similarly as in the proof of Theorem $1.2(\mathrm{i})$. Here we start with a homogeneous polynomial $P_{0} \in \mathbb{Q}\left[x_{2}, \ldots, x_{d+1}\right]$ which is a sum of $d+1$, but not of $d$, squares in $R\left(x_{2}, \ldots, x_{d+1}\right)$, for any real closed field $R$. For the existence of such $P_{0}$, the essential case is $d=3$. This was first established in a beautiful and difficult paper by Cassels, Ellison and Pfister [CEP], who showed that the Motzkin polynomial is not a sum of three squares of rational functions. See $[\mathrm{BCR}, 6.4 .8,6.4 .20]$ for how to deduce the case of general $d \geq 3$ from this.

\section{ACKNOWLEDGEMENT}

We would like to thank the anonymous referee for careful reading and for a very helpful list of remarks and suggestions.

\section{REFERENCES}

[ABR] C. Andradas, L. Bröcker, J.M. Ruiz: Constructible Sets in Real Geometry. Ergeb. Math. Grenzgeb. 33. Berlin Heidelberg New York: Springer Verlag, 1996. MR 98e:14056

[BCR] J. Bochnak, M. Coste, M.-F. Roy: Real Algebraic Geometry. Ergeb. Math. Grenzgeb. 36. Berlin Heidelberg New York: Springer-Verlag, 1998. MR 2000a:14067.

[CaRz] A. Campillo, J.M. Ruiz: Some remarks on pythagorean real curve germs, J. Algebra 128, 271-275 (1990). MR 91b:14072

[CEP] J.W.S. Cassels, W.J. Ellison, A. Pfister: On sums of squares and on elliptic curves over function fields. J. Number Theory 3, 125-149 (1971). MR 45:1863

[CDLR] M.D. Choi, Z.D. Dai, T.Y. Lam, B. Reznick: The Pythagoras number of some affine algebras and local algebras, J. reine angew. Math. 336, 45-82 (1982). MR 84f:12012

[Fe1] J.F. Fernando: Positive semidefinite germs in real analytic surfaces, Math. Ann. 322, 49-67 (2002). MR 2003b:14069

[Fe2] J.F. Fernando: On the Pythagoras numbers of real analytic rings, J. Algebra 243, 321-338 (2001). MR 2002g:13051

[Fe3] J.F. Fernando: Analytic surface germs with minimal Pythagoras number, Preprint Univ. Complutense, Madrid 2002.

[Fe4] J.F. Fernando: Sums of squares in real analytic rings, Trans. Am. Math. Soc. 354, 19091919 (2002). MR 2003b:14070

[FeRz1] J.F. Fernando, J.M. Ruiz: Positive semidefinite germs on the cone, Pacific J. Math. 205, 109-118 (2002). MR 2003f: 14066

[FeRz2] J.F. Fernando, J.M. Ruiz: On the Pythagoras numbers of real analytic set germs (in preparation).

[JP] T. de Jong, G. Pfister: Local Analytic Geometry. Adv. Lect. Math. Braunschweig/Wiesbaden: Vieweg, 2000. MR 2001c:32001

[Mt] H. Matsumura: Commutative Algebra, 2nd edition. London Amsterdam Tokyo: Benjamin, 1980. MR 82i:13003

[Ng] M. Nagata: Local Rings. New York London: John Wiley \& Sons, 1962. MR 27:5790

[Or] J. Ortega: On the Pythagoras number of a real irreducible algebroid curve. Math. Ann. 289, 111-123 (1991). MR 92a:14065

[Pf] A. Pfister: Quadratic Forms with Applications to Algebraic Geometry and Topology. London Math. Soc. Lect. Notes 217, Cambridge, 1995. MR 97c:11046

[PD] A. Prestel, C.N. Delzell: Positive Polynomials. Monographs in Mathematics. Berlin Heidelberg New York: Springer Verlag, 2001. MR 2002k:13044

[Qz] R. Quarez: Pythagoras numbers of real algebroid curves and Gram matrices. J. Algebra 238, 139-158 (2001). MR 2002g:14086

[Rz1] J.M. Ruiz: On Hilbert's 17th problem and real Nullstellensatz for global analytic functions. Math. Z. 190, 447-459 (1985). MR 87b:32010

[Rz2] J.M. Ruiz: Sums of two squares in analytic rings. Math. Z. 230, 317-328 (1999). MR 2000b:58068 
[Sch1] C. Scheiderer: Sums of squares of regular functions on real algebraic varieties, Trans. Am. Math. Soc. 352, 1039-1069 (1999). MR 2000j:14090

[Sch2] C. Scheiderer: On sums of squares in local rings, J. reine angew. Math. 540, 205-227 (2001). MR 2002j:13031

[Sch3] C. Scheiderer: Sums of squares on real algebraic curves, Math. Z. (to appear)

[Sch4] C. Scheiderer: Sums of squares on compact real algebraic surfaces (in preparation).

Departamento de Álgebra, Facultad Ciencias Matemáticas, Universidad Complutense DE MADRID, 28040 MAdRID, Spain

E-mail address: josefer@mat.ucm.es

Departamento de Geometría y Topología, Facultad Ciencias Matemáticas, UniversiDad Complutense de Madrid, 28040 Madrid, Spain

E-mail address: jesusr@mat.ucm.es

Institut für Mathematik, Fakultät 4, Universität Duisburg, 47048 Duisburg, GerMANY

E-mail address: claus@uni-duisburg.de 